\title{
Hollow cathode life-time prediction from low work function surface coverage evolution
}

\author{
M. Coletti ${ }^{1}$ and S. B. Gabriel ${ }^{2}$ \\ University of Southampton, Southampton, United Kingdom, SO17 1BJ
}

\begin{abstract}
In this paper a procedure to update the plasma parameters relatively to changes of the hollow cathode surface work function has been developed. This procedure has been validated with experimental results and then coupled with the deposition/desorption model already developed by the authors. The evolution of the surface of the cathode tested for 28,000 hours by Sarver-Verhey has been simulated and from the data obtained an end of life criterion has been developed. Then using this criterion the ELT NSTAR discharge cathode has been simulated for various throttle conditions deriving the lifetime at THO $(200,000 \mathrm{~h})$, TH8 $(100,000 \mathrm{~h})$ and TH15 $(25,000 \mathrm{~h})$. The lifetimes found are in agreement with the theoretical expectation. The lifetime of the ELT NSTAR cathode under the throttling conditions used during the Deep Space 1 Spare Ion Engine life test has been found to be in excess of 30,000 hours and in particular 100,000 or more if the cathode is run at TH8 or lower from 30,000 hours onward.
\end{abstract}

\section{Nomenclature}

$=$ area

$=$ lifetime temperature constant

$=$ area view factor

$=$ current

$=$ current density

$=$ plasma characteristics multiplicative factor

$=$ Boltzmann constant

$=$ molecular mass

$=$ mass

$=$ mass flow rate

$=$ molecules flow rate per unit are per unit time

$=$ number density

$=$ electron charge

$=$ irradiated power

$=$ universal gas constant

$=$ radial coordinate

$=$ surface

$=$ temperature

$=$ time

$=$ voltage

$=$ thermal velocity

$=$ coordinate along the insert length

$=$ sputtering coefficient

= breakdown voltage amplification factor

$=$ life increase factor

\footnotetext{
${ }^{1}$ PhD Student, Astronautic Research Group, School of Engineering Sciences, Coletti@soton.ac.uk, AIAA student member.

${ }^{2}$ Prof. of and Head of the Astronautic Research Group, School of Engineering Sciences, sbg2@soton.ac.uk, AIAA member.
} 


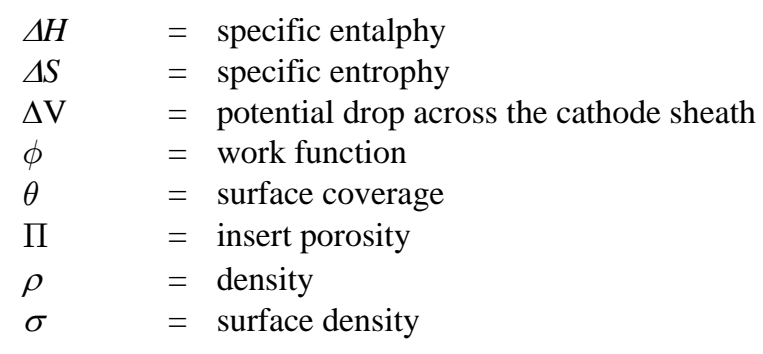

\section{Subscripts}

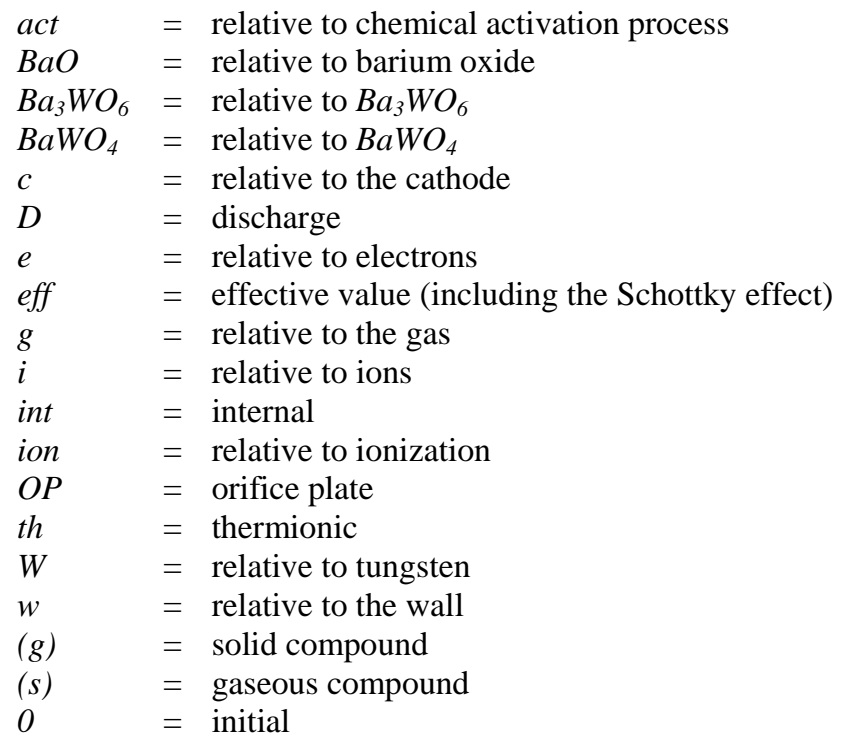

\section{Superscript}

$\begin{array}{ll}\text { bomb } & =\text { relative to bombardment } \\ t h & =\text { thermal } \\ + & =\text { depostion } \\ - & =\text { desorption }\end{array}$

\section{Introduction}

$\mathrm{H}^{\circ}$ OLLOW cathodes are a key component in the electric propulsion field. They are used as electron sources and neutralizers inside ion thrusters and Hall's effect thrusters and in the next future probably as stand alone microthrusters ${ }^{1,2}$ therefore their lifetime and performance are key elements in all the application mentioned above.

Hollow cathodes developed by NASA have to date demonstrated a lifetime of 30,000 hours in ground testing ${ }^{3}$ and 1,600 hours in orbit, however due to the requirements of longer lifetimes for deep space mission a large amount of efforts has been spent in lifetesting, characterization and modelling of hollow cathodes to ultimately estimate their lifetime ${ }^{3-14}$.

During normal operation of the cathode the work function of the emitter surface is lowered from the typical value of the emitter material (for tungsten $\phi=4.5 \mathrm{eV}$ ) down to about $2 \mathrm{eV}$ thanks to the evaporation of $\mathrm{BaO}$ from the emitter core and its subsequent deposition on the emitting surface resulting in the formation of low work function compounds.

During cathode life the evaporation rate of $\mathrm{BaO}$ from the insert tends to decrease due to the gradual depletion of $\mathrm{BaO}$, hence the low work function deposition rates will decrease as well, resulting in a reduction of the area covered by low work function compounds and in an increase of the overall work function. This process leads to a reduction of the thermionic current from the emitter that, in turn, will force the cathode to operate at a higher temperature 
increasing the $\mathrm{BaO}$ depletion rate. This process will continue up to the point where the cathode is no longer able to operate.

The lifetime of the cathode is then mainly dependent on the cathode operating temperature and how this affects the chemistry of the low work function material deposition and its rate of loss.

It is then clear how the understanding of the evaporation and deposition process involving $\mathrm{BaO}$ and of the processes that results in low work function desorption from the surface is key in predicting the lifetime of the cathode.

Barium oxide evaporation and depletion from the insert has been already studied and modelled by the authors ${ }^{15-}$ ${ }^{17}$ starting from the chemistry of the $\mathrm{BaO}-\mathrm{CaO}-\mathrm{Al}_{2} \mathrm{O}_{3}$ system. The model has also been validated comparing the numerical results with the experimental measurements finding satisfactory agreement ${ }^{16,17}$.

Deposition and desorption of low work function compounds has been studied as well ${ }^{18,19}$ and the main results found will be reported in Sec. II.

In this paper an easy semi empirical model to predict the change in the plasma characteristics due to the changes in the low work function surface coverage will be presented. This model will be validated using experimental results and then will be coupled with the deposition/desorption model.

From the results so obtained an end-of-life criterion will be developed and the lifetime of the NSTAR cathode will be predicted for various throttling conditions.

\section{Deposition and desorption of low work fucntion compound ${ }^{18}$}

Starting from the chemical data published by Resulhina ${ }^{20}$, Chang $^{21}$, Phillips ${ }^{22}$, Kreidler $^{23}$, Bondarenko ${ }^{24}$ and on some of the results found by Langmuir ${ }^{25-29}$ and easy deposition and depletion model has been developed ${ }^{18,19}$.

First of all, based on the experimental measurements reported in Ref 24, the main low work function compound has been found to be $\mathrm{Ba}_{3} \mathrm{WO}_{6}$.

The work function of this compound is ${ }^{24}$

$$
\phi=1.33+6.09 \cdot 10^{-4} T
$$

This work function is plotted below together with the average hollow cathode work function measured at NASA Jet Propulsion Laboratory ${ }^{6}$.

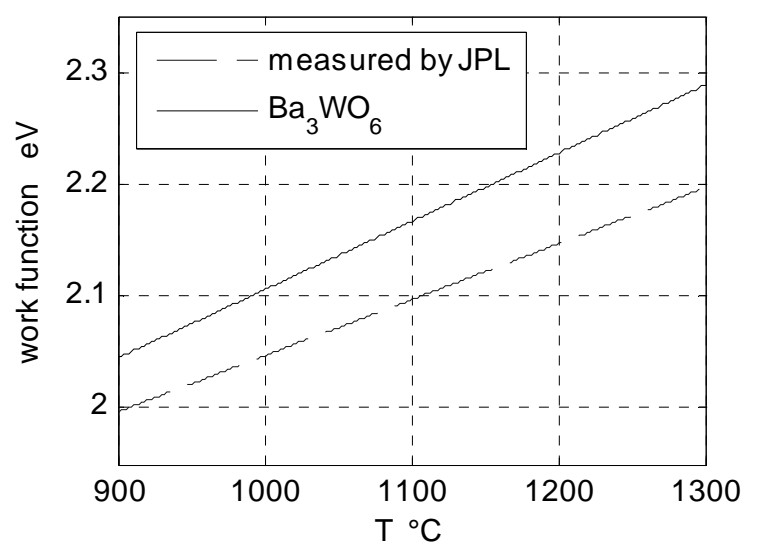

Fig. 1 Comparison between the measured HC work function and the one relative to $\mathrm{Ba}_{3} \mathrm{WO}_{6}$

It can be noted how the two trends are very close presenting a maximum difference of about $4 \%$ at 1300 ${ }^{\circ} \mathrm{C}$.

From the assumption that $\mathrm{Ba}_{3} \mathrm{WO}_{6}$ is the main low work function compound the $4 \mathrm{BaO}: 1 \mathrm{CaO}: 1 \mathrm{Al}_{2} \mathrm{O}_{3}$ ratio commonly used in $\mathrm{HC}$ has been qualitatively explained. In fact looking at the $\mathrm{BaO}-\mathrm{WO}_{3}$ and $\mathrm{CaO}-$ $\mathrm{WO}_{3}$ phase diagrams a strong similarity between the compounds formed by calcium oxide and barium oxide has been found and partial substitution of $\mathrm{BaO}$ with $\mathrm{CaO}$ has also been found to occur ${ }^{21-23}$.

The only difference between barium-tungsten and calcium-tungsten compounds is that the compounds formed by $\mathrm{CaO}$ have a much higher resistance to temperature.

The work functions of calcium-tungsten compounds have not been found in the literature but, considering the similarities in atomic characteristics and work function between calcium and barium, can be assumed that the work functions of the solid solutions $\mathrm{Ba}_{3-\mathrm{x}} \mathrm{Ca}_{\mathrm{x}} \mathrm{WO}_{6}$ will not be sensibly different from the one relative to $\mathrm{Ba}_{3} \mathrm{WO}_{6}$.

From these observations the presence of $\mathrm{CaO}$ inside the impregnate has been justified as follows. Barium oxide is a compound that evaporates at relatively low temperatures and once evaporated, depositing on the emitter surface and reacting with tungsten creates low work function compounds (mainly $\mathrm{Ba}_{3} \mathrm{WO}_{6}$ ); $\mathrm{CaO}$ instead has a much lower evaporation rate whereas its compounds can resist to much higher temperatures than the one formed by barium oxide. Therefore a partial substitution of $\mathrm{BaO}$ with $\mathrm{CaO}$ will generate high temperature resistant low work function compounds. 
The function of $\mathrm{BaO}$ is, thanks to its relatively high evaporation rates, to provide the "base material" needed to build as many low work function compounds as possible; the function of $\mathrm{CaO}$ is, substituting a small fraction of $\mathrm{BaO}$, to give to these compounds resistance to temperature. Considering all this the usual 4:1 ratio between $\mathrm{BaO}$ and $\mathrm{CaO}$ can be qualitatively explained.

It has been found that $\mathrm{Ba}_{3} \mathrm{WO}_{6}$ is formed in two steps, firstly $\mathrm{BaO}$ react with $\mathrm{WO}_{3}$ creating $\mathrm{BaWO}_{4}$, a high temperature resistant, high work function compound, and then $\mathrm{BaWO}_{4}$ reacts with other $\mathrm{BaO}$ to form $\mathrm{Ba}_{3} \mathrm{WO}_{6}$.

The reaction involving $\mathrm{Ba}_{3} \mathrm{WO}_{6}$ and $\mathrm{BaWO}_{4}$ are reported below ${ }^{20,30}$

Table 1 Low work function compounds reactions

\begin{tabular}{|c|c|c|}
\hline Reaction & $\Delta H \mathrm{eV}$ & $\Delta S \mathrm{~kJ} / \mathrm{mol}$ \\
\hline $\mathrm{Ba}_{3} \mathrm{WO}_{6(s)} \Longleftrightarrow 2 \mathrm{BaO}_{(\mathrm{g})}+\mathrm{BaWO} \mathrm{O}_{4(s)}$ & 11.39 & 320.11 \\
\hline $\mathrm{BaWO}_{4(s)} \Longleftrightarrow \mathrm{BaO}_{(\mathrm{g})}+\mathrm{WO}_{3(s)}$ & 12.34 & 137.98 \\
\hline
\end{tabular}

Deposition of barium oxide on the emitter surface is the results of the reactions reported in Table 1 when these proceed from right to left whereas thermal desorption occurs when they proceed from left to right.

To predict the exact behaviour of deposition with time the forward and backward reactions constants are needed. Unfortunately these data has not been found in the literature; the only data available are relative to the equilibrium constant of the reactions.

These equilibrium constants are very small (respectively $10^{-26}, 10^{-39}$ ) meaning that the reactions are strongly shifted to the left and hence that $\mathrm{BaO}$ tends to react very quickly with $\mathrm{WO}_{3}$ to form $\mathrm{BaWO}_{4}$ and then $\mathrm{Ba}_{3} \mathrm{WO}_{6}$.

From this consideration the deposition rate have been expressed as

$$
\dot{N}_{B a W O_{4}}^{+}=\theta_{W}(1-\Pi) \frac{\dot{m}_{B a O}}{m_{B a O}} \quad ; \quad \dot{N}_{B a_{3} W O_{6}}^{+}=\frac{1}{2} \theta_{B a W O_{4}}(1-\Pi) \frac{\dot{m}_{B a O}}{m_{B a O}}
$$

where $\theta_{W}$ and $\theta_{\mathrm{BaWO}_{4}}$ are respectively the fraction of the surface covered by tungsten and by $\mathrm{BaWO}_{4}$.

The desorption rates relative to thermal decomposition have been modelled as

$$
\begin{gathered}
\dot{N}_{B a_{3} W O_{6}}^{-t h}=\frac{1}{2} \theta_{B a_{3} W O_{6}}(1-\Pi) \frac{e^{\left(-\frac{\Delta H}{k_{b} T}+\frac{\Delta S}{R}\right) \frac{1}{2}}}{m_{B a O}} \frac{10^{5}}{\sqrt{\frac{2 \pi R T}{M_{B a O}}}} \\
\dot{N}_{B a W O_{4}}^{-t h}=\theta_{B_{a W O_{4}}}(1-\Pi) \frac{e^{\left(-\frac{\Delta H}{k_{b} T}+\frac{\Delta S}{R}\right)}}{m_{B a O}} \frac{10^{5}}{\sqrt{\frac{2 \pi R T}{M_{B a O}}}}
\end{gathered}
$$

whereas the ones relative to ion sputtering due to the ion flux from the hollow cathode plasma have been expressed as

$$
\begin{gathered}
\dot{N}_{B^{-b a W O_{4}}}^{-b o m b}=n_{i} v_{t h}(1-\Pi) \theta_{B a W O_{4}} e^{\frac{-E_{a c t}}{\alpha \Delta V}} \\
\dot{N}_{B a_{3} W O_{6}}^{-b o m b}=n_{i} v_{t h}(1-\Pi) \theta_{B a_{3} W O_{6}} e^{\frac{-E_{a c t}}{\alpha \Delta V}}
\end{gathered}
$$

where $n_{i}$ and $\Delta V$ are the plasma particle density and the voltage drop between plasma and cathode, where the activation energy has been approximated with the free Gibbs energy of the reactions in Table 1 and where $\alpha$ is ${ }^{25}$

$$
\alpha=4 m_{c} m_{g}\left[\frac{m_{g}-m_{c}}{\left(m_{g}+m_{c}\right)^{2}}\right]^{2}
$$

and $m_{c}$ and $m_{g}$ are respectively the mass of the impacting ion and of the cathode molecule. 
In Ref 18, 19 the deposition and desorption problem has been solved focusing only on $\mathrm{Ba}_{3} \mathrm{WO}_{6}$ whereas here the evolution of both $\mathrm{Ba}_{3} \mathrm{WO}_{6}$ and $\mathrm{BaWO}_{4}$ will be calculated.

We have that the surface coverage of these two compounds can be represented with a system of linear differential equation

$$
\begin{gathered}
\dot{\theta}_{B a W O_{4}}=\frac{m_{B a O}}{\sigma_{B a O}}\left[\dot{N}_{B a W O_{4}}^{+}+\left(\dot{N}_{B a_{3} W O_{6}}^{-t h}+\dot{N}_{B a_{3} W O_{6}}^{-b o m b}\right)-\left(\dot{N}_{B a W O_{4}}^{-t h}+\dot{N}_{B a W O_{4}}^{-b o m b}\right)\right] \\
\dot{\theta}_{B a_{3} W O_{6}}=\frac{m_{B a O}}{\sigma_{B a O}}\left[\dot{N}_{B a_{3} W O_{6}}^{+}-\left(\dot{N}_{B a_{3} W O_{6}}^{-t h}+\dot{N}_{B a_{3} W O_{6}}^{-b o m b}\right)\right]
\end{gathered}
$$

This system has been solved analytically and from the values of $\theta_{B a W O_{4}}$ and $\theta_{B a_{3} W O_{6}}$ together with the knowledge of the $\mathrm{BaO}$ concentration inside the pores the work function of the surface has been calculated.

\section{Plasma Parameter Update}

As it can be seen in the sections above the knowledge of the hollow cathode plasma parameters and of the insert temperatures are essential to estimate the desorption rates and hence the surface coverage of low work function compounds.

To do so the ideal solution will be to have a plasma model that starting from inputs like total cathode current, mass flow rate and cathode dimension will be able to calculate the ion and electron temperature and density profiles along the insert length together with the voltage fall and insert temperature profiles.

The only model that at present is able to fit these requirements is the plasma model developed at JPL ${ }^{6-9}$;

Since we can not have access to this code an alternative way to obtain such plasma parameters will be developed.

We will assume quasi-neutrality $\left(n_{i}=n_{e}=n\right)$, temperature equilibrium between the heavy particles and the wall $\left(T_{i}=T_{w}\right)$ and we will assume that given a hollow cathode to simulate with the model described above, the plasma parameters at the beginning of life are known by measurements.

We will also assume that the dependency of the plasma parameters from time and space can be divided in two separate functions so that is possible to write

$$
\begin{aligned}
\Delta V(t, z) & =k_{\Delta V}(t) \Delta V(0, z) \\
n(t, z) & =k_{n}(t) n(0, z) \\
T_{e}(t, z) & =k_{T_{e}}(t) T_{e}(0, z) \\
T_{w}(t, z) & =k_{T_{w}}(t) T_{w}(0, z)
\end{aligned}
$$

meaning that the shape of the plasma parameters trend will remain the same with time whereas their values will be shifted up or down by a multiplicative factor.

It could be argued that the ion density profile is linked to the thermionic emission profile hence a change in the surface coverage distribution will affect consequently the particle density trend invalidating the last of the assumptions above.

To judge this a comparison between the plasma electron density profile taken from the experimental data in Ref 9 relative to the NSTAR cathode functioning at $12 \mathrm{~A}$, and the emitted electron density profile is shown in Fig. 2 where $\mathrm{z}$ is non-dimensional cathode abscissa and where the emitted electron density is calculated assuming that the electrons leave the surface with their thermal speed 


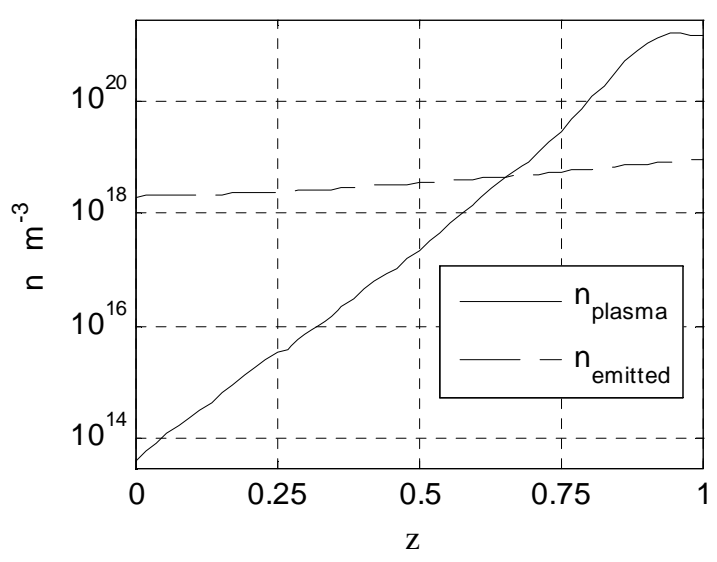

Fig. 2 Comparison between plasma electron density ${ }^{9}$ and emitted electron density

upstream region.

Nevertheless this hypothesis has been often used along the whole length of the cathode producing results that closely match the experimental measurements ${ }^{6-9}$.

Considering what has been said above and considering that the most important region is the downstream end of the cathode we can conclude that the assumption of constant shape of the electron density profile during the cathode life is acceptable.

Once this assumption has been accepted it might extended to the plasma voltage drop and to the electron and wall temperature since all these quantities are strictly interdependent.

We will also assume that the electron temperature will not vary sensibly due to the change in the surface coverage as its value has been shown to have small variations over a wide range of cathode operating conditions ${ }^{32}$.

From all the assumptions above the update of the plasma parameters has been reduced to the calculation of three time-dependent multiplicative parameters $\left(k_{\Delta V}, k_{n}, k_{T_{w}}\right)$.

To derive these parameters the power balance at the cathode surface and the total emitted current conservation will be imposed

$$
\begin{gathered}
\int J_{t h}\left(\phi_{e f f}+\frac{5 k T_{e}}{2 q}\right) d A= \\
\int J_{e}\left(\phi_{e f f}+\frac{5 k T_{w}}{2 q}\right) d A+ \\
+\int J_{i}\left(E_{\text {ion }}+\Delta V-\phi_{\text {eff }}\right) d A+f q_{r} \\
\int\left(J_{t h}+J_{i}-J_{e}\right) d A=I_{D}
\end{gathered}
$$

During the simulation of the hollow cathode the plasma parameters will be updated at fixed time steps. The distance between two consecutive time steps will be defined to obtain accurate simulations with reasonable computing times and will be of the order of 10 hours.

At every update Eq. (13), (14) will be solved assuming constant wall temperature deriving the values of $k_{\Delta V}$ and $k_{n}$. It might happen that a solution of these equations cannot be found. This means that the insert temperature is too low to provide enough thermionic emission to meet the total current emission requirements set by the power supply. In this case the insert temperature will be increased by $10^{\circ} \mathrm{C}$ steps until a solution of Eq. (13) and (14) can be found.

\section{Plasma Parameter Update procedure Validation}

Prior to start using the surface coverage model reported in Sec. II the plasma parameter update process must be validated. 
In this section the procedure developed in Sec. III will be tested with the data available in the literature relative to the same cathode that will be simulated later.

In Ref 9 and 33 the data relative to NSTAR cathode electron temperature, ion/electron number density and plasma voltage have been reported relatively to discharge currents of 7.6 (TH8), 12 and 13.5 A (TH13).

To simulate the NSTAR cathode the plasma characteristics relative to the throttle level TH12, TH5 and TH0 are needed; these data will be derived using the parameter update process described in Sec. III assuming that the ion/electron density is constant among all the TH level.

If we compare the densities relative to 7.6 A, $12 \mathrm{~A}$ and to $13.5 \mathrm{~A}$ of discharge current we can see how these three trends are quite close even if from TH15 to TH8 the discharge current drops to almost half of its value.

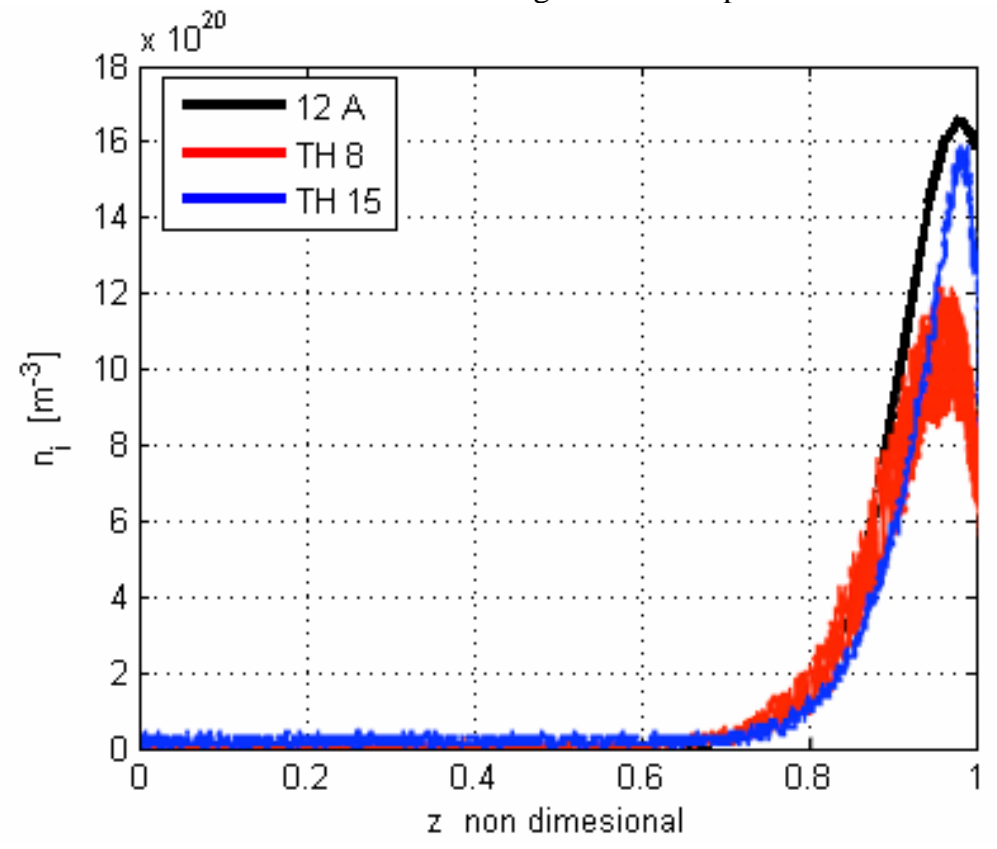

Fig. 3 Ion density at TH15, TH8 and at 12 A of discharge current ${ }^{9,33}$

Since the ion density relative to 12 ampere of discharge current is the highest between the measured ones if we assume that the ion density is constant for all the THs and equal to the one relative to 12A we will most probably overestimate $n_{i}$ hence overestimating the ion sputtering desorption rates finally producing conservative estimates of the surface coverage evolution and of the cathode lifetime.

Once the hypothesis of constant ion/electron density has been done the trend of $\Delta V$ and $T_{e}$ can be derived from the measured data relative to $12 \mathrm{~A}$. 


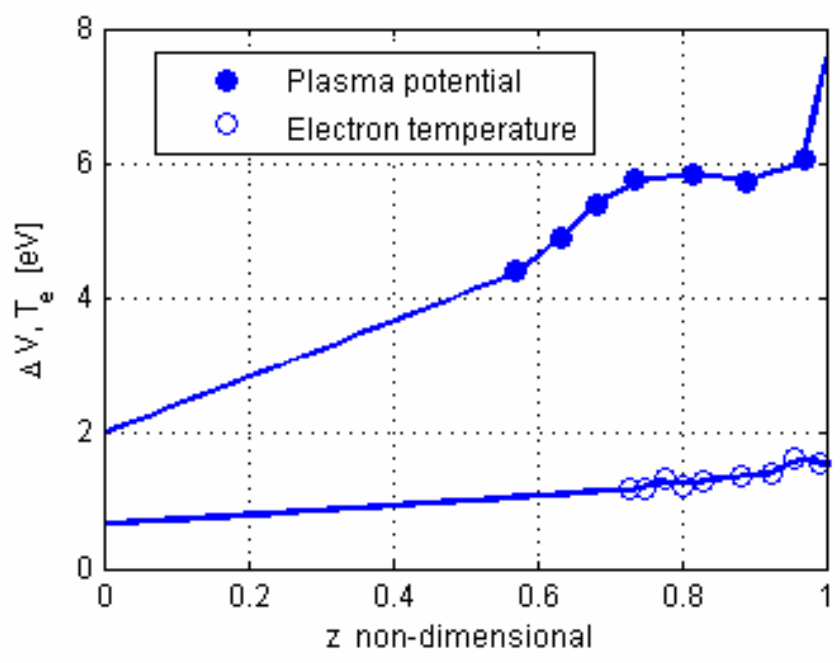

Fig. 4 Measure plasma potential and electron temperature (circles) for $12 \mathrm{~A}$ and assumed fitting profiles (solid lines) ${ }^{9}$

Using the hypothesis of constant $n_{i}$ and the profiles of $\Delta V$ and $T_{e}$ shown above, solving Eq. (13) and (14), the plasma voltage and electron temperature of every TH level can be derived.

Below is a comparison between the calculated values of $\Delta V$ and $T_{e}$, and the experimental ones for TH15 and TH8 reported in Ref. 33

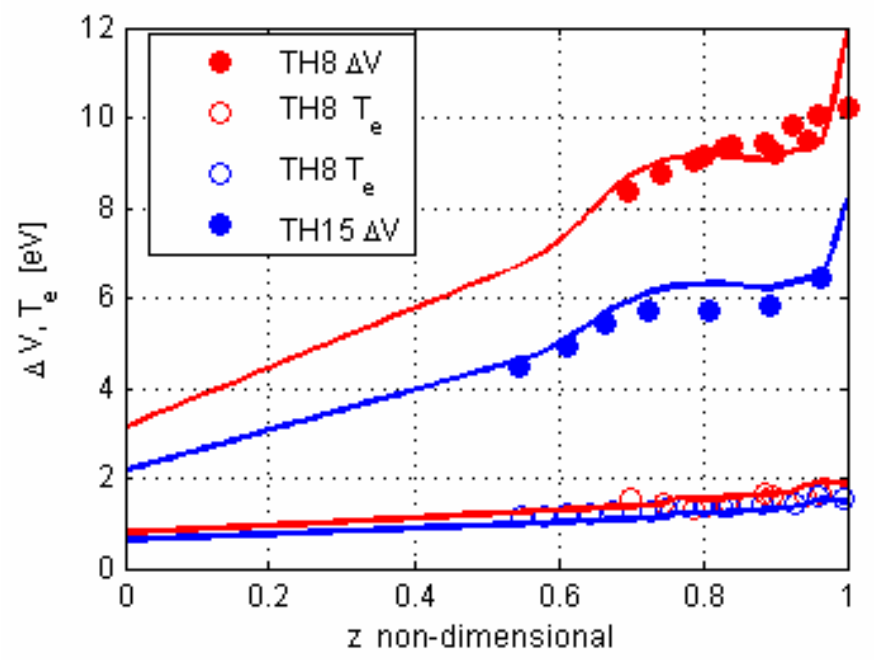

Fig. 5 Comparison between calculated and measure plasma parameters for TH8 and TH15

As it can be seen the computed data show a very good agreement with the measured one proving the goodness of the plasma parameters update procedure.

Once this procedure has been validated the plasma parameters of the remaining throttle levels can be calculated. The results are reported below. 


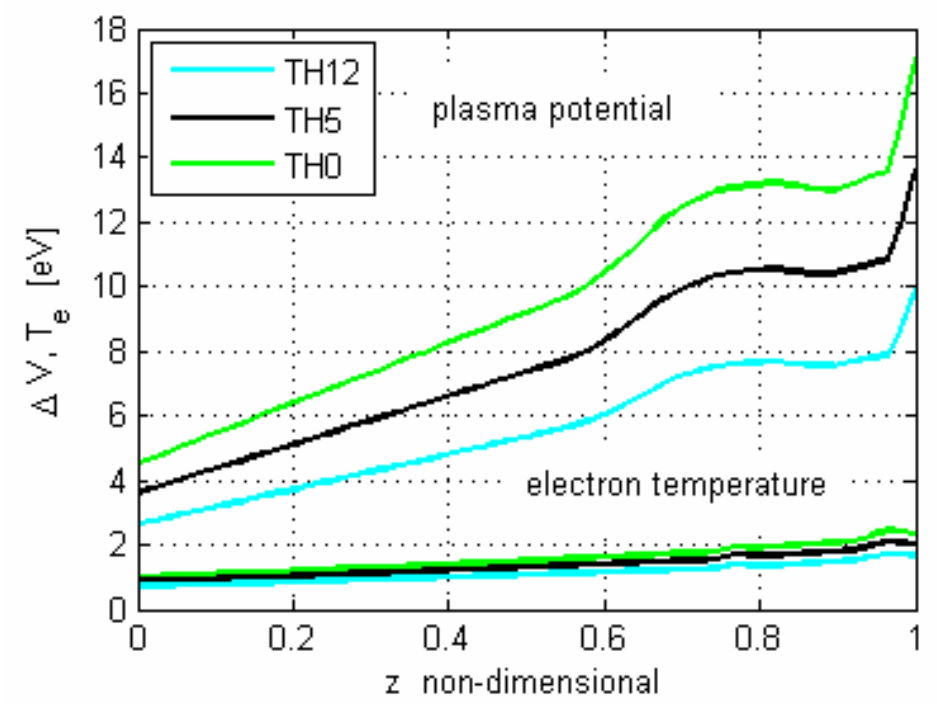

Fig. 6 Calculated plasma voltage and temperature profiles for TH12, TH5 and TH0

As it can be seen the plasma voltage tends to increase as the discharge current decrease. This can be explained looking at the thermionic emitted current for each throttle level and comparing them with the discharge currents.

Table 2 - Emitted and discharge currents

\begin{tabular}{ccc}
\hline \hline TH level & Emitted currents [A] & Discharge Current [A] \\
\hline 0 & 9.07 & 4.9 \\
5 & 13.4 & 6.9 \\
8 & 16.8 & 7.6 \\
12 & 22.8 & 9.9 \\
15 & 32.3 & 13.5 \\
\hline \hline
\end{tabular}

The cathode insert always emits currents that are in excess of the desired discharge current value, this means that for every $\mathrm{TH}$ the electron flux must be modulated by the interaction of electron temperature and voltage fall so that the total current flowing into the cathode is equal to the value fixed by the power supply (discharge current).

Since the variations in electron temperature are small and the electron density is assumed to be constant, the plasma voltage will be the parameter that mainly influences the electron flux.

Since the electron flux needed at lower discharge currents is smaller than at the higher ones and since the higher the plasma voltage the lower is the electron current, the plasma voltage must be high at low THs and low at high THs hence explaining the trend shown in Fig. 5, 6.

\section{28,000 hours cathode simulation}

The cathode reported in Ref 4, 5 by Sarver-Verhey has been tested for 28,000 hours at $12 \mathrm{~A}$ of discharge current.

We will assume that its insert temperature profile and plasma parameters are the same as those reported for the NSTAR cathode at 12A.

Using this assumption the cathode surface has been simulated for 28,000 hours. During this simulation the plasma parameters have been updated every 50 hours assuming the electron temperature to be constant during the whole simulation of the cathode.

This assumption can be justified noting that the plasma temperature has shown small variations over a wide range of discharge currents and emitted currents (Fig. 4-6) whereas the plasma voltage has shown to be strongly dependant on the emission and discharge currents.

Regarding the ion density we can note that if the thermionically emitted current drops below the discharge current value the ion flux from the plasma to the surface must become big enough to overcome the electron flux providing the additional current needed to reach the discharge value. Hence a reduction in the emitted current due to 
a reduction in the surface coverage will result in an increase of the ion density (to increase the ion current) and in an increase of the plasma voltage to decrease the electron current that, otherwise, since quasi-neutrality has been assumed, will increase proportionally to the ion flux.

The computed surface coverage relative to $\mathrm{Ba}_{3} \mathrm{WO}_{6}$ and $\mathrm{BaWO}_{4}$ and the work function evolution are reported below

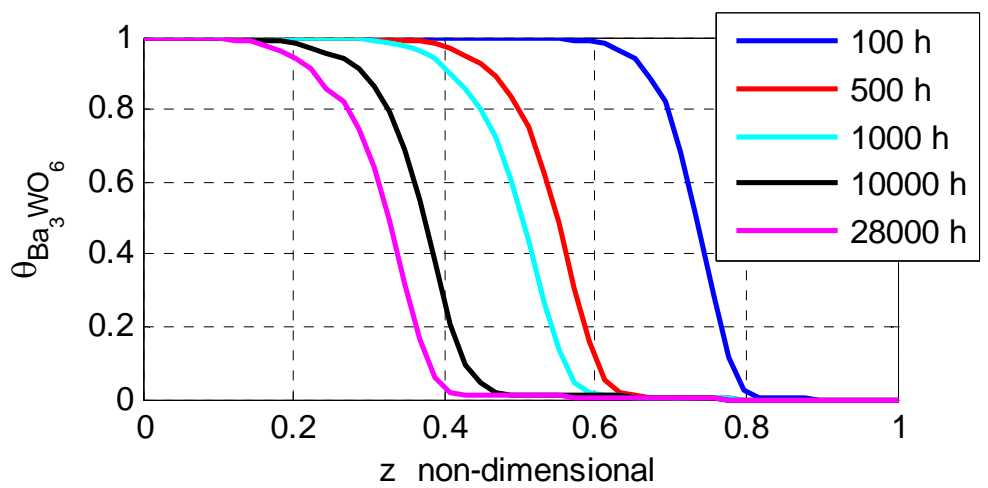

Fig. $7 \mathrm{Ba}_{3} \mathrm{WO}_{6}$ surface coverage - 12A

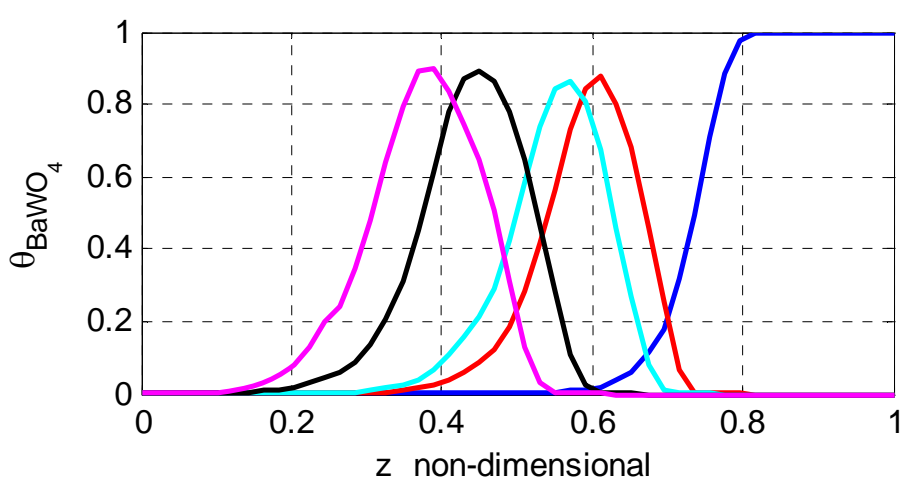

Fig. $8 \mathrm{BaWO}_{4}$ surface coverage - 12A

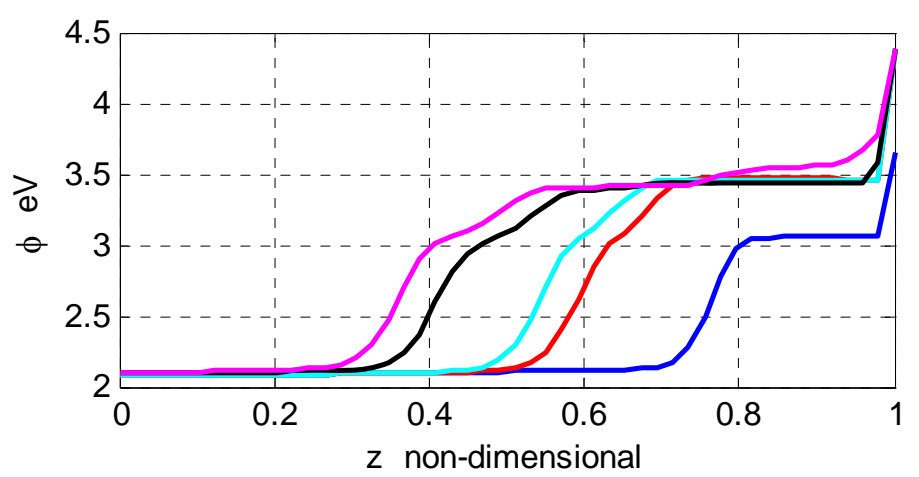

Fig. 9 Surface work function - 12A

As it can be noted in the figures above the low work function compounds depletion starts at the downstream end of the cathode and then slowly moves upstream. This is due to the fact that the sputtering desorption rate is proportional to ion density and plasma voltage and that these two parameters are maximum at the downstream end of the cathode.

It must also be noted how the time needed to remove $\mathrm{BaWO}_{4}$ from the surface is longer than the one needed to remove $\mathrm{Ba}_{3} \mathrm{WO}_{6}$ mitigating the work function increase. 
Looking at Fig. 9 we can see how even when all the $\mathrm{Ba}_{3} \mathrm{WO}_{6}$ and $\mathrm{BaWO}_{4}$ have been removed from the surface the work function is still below the bare tungsten value. This is due to the presence of barium oxide in the pores that contribute to the overall emission hence lowering the average work function value of the surface.

We can now analyze the evolution of the plasma voltage and ion/electron density with time. The values of the parameters $k_{n}$ and $k_{\Delta V}$ as defined in Eq. (11) are reported in Fig. 10.

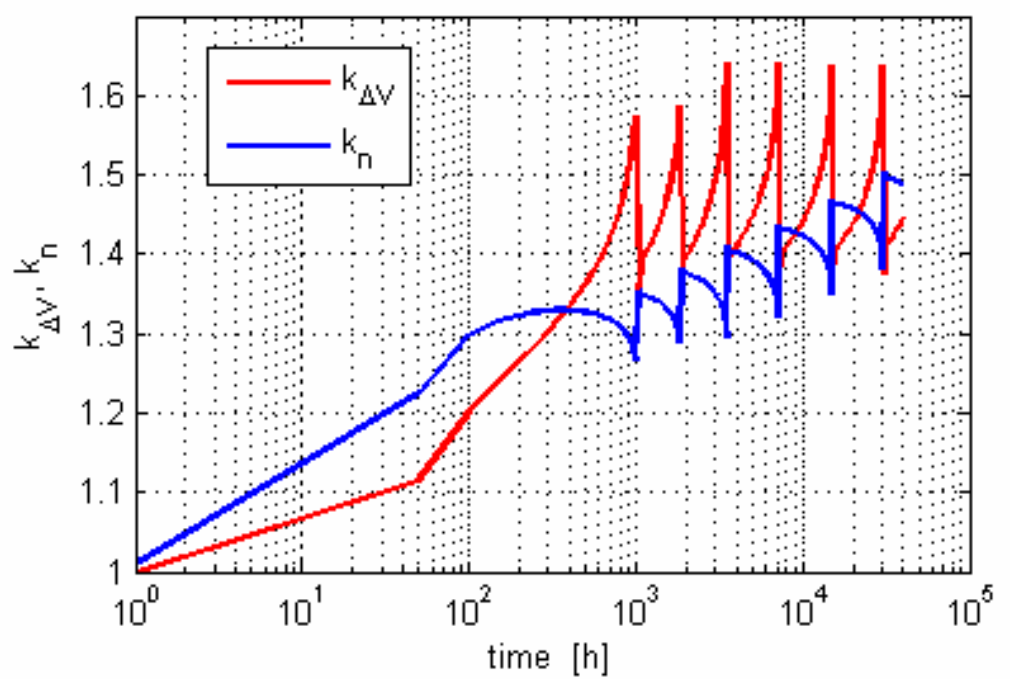

Fig. $10 \quad k_{n}$ and $k_{\Delta v}$ trend with time $-12 A$

The periodic oscillations in the values of these constants are the effect of the hypotheses made in Sec. III. In fact we have assumed the insert temperature profile to be constant with time whereas changes in the surface coverage will definitely affect its value. The insert temperature has been updated only when no solution to Eq. (13) and (14) can be found meaning that the thermionic emission from the surface is too low to reach the required discharge current. The update procedure consists in increasing the insert temperature value by steps of $10^{\circ} \mathrm{C}$ until a solution for Eq. (13) and (14) can be found. In the real functioning of the cathode the insert temperature will

vary gradually together with the plasma voltage and ion density. Forcing the temperature to increase only when the cathode has reached a critical condition (emitted current too low to reach the required discharge current as for example after 1000 hours in Fig. 10) will produce an overestimation of the plasma voltage since the cathode, not being able to get more current from the thermionic emission increasing the insert temperature, will try to increase the ion current and to decrease the electron one to meet the required discharge current hence increasing both the value of $\Delta V$ and $n$. Since the plasma voltage is overestimated this will produce conservative estimates on the surface coverage and ultimately on the cathode lifetime.

When a critical condition is going to be reached the plasma voltage will increase and the ion density will decrease reducing the value of the electron current so that the total discharge current can be obtained by the sum of $I_{t h}$ and $I_{i}$. This explains the behavior of $k_{\Delta V}$ and $k_{n}$ just before the "steps" in Fig. 10.

When the critical condition is finally reached the insert temperature is suddenly increased increasing the thermionic emitted current hence allowing the cathode to reduce the plasma potential and to increase the particle density bringing them to a value close to the one they had before the critical condition was approached. This explains the trend of $k_{\Delta V}$ and $k_{n}$ just after the "steps" in Fig. 10.

Since the ion current is directly proportional to the ion number density the electron current will be reduced mainly increasing the plasma voltage hence justifying the bigger variation in $k_{\Delta V}$ than in $k_{n}$ as reported in the figure above.

Once the values of plasma voltage particle density and work function are known the currents flowing at the cathode surface can be calculated. 


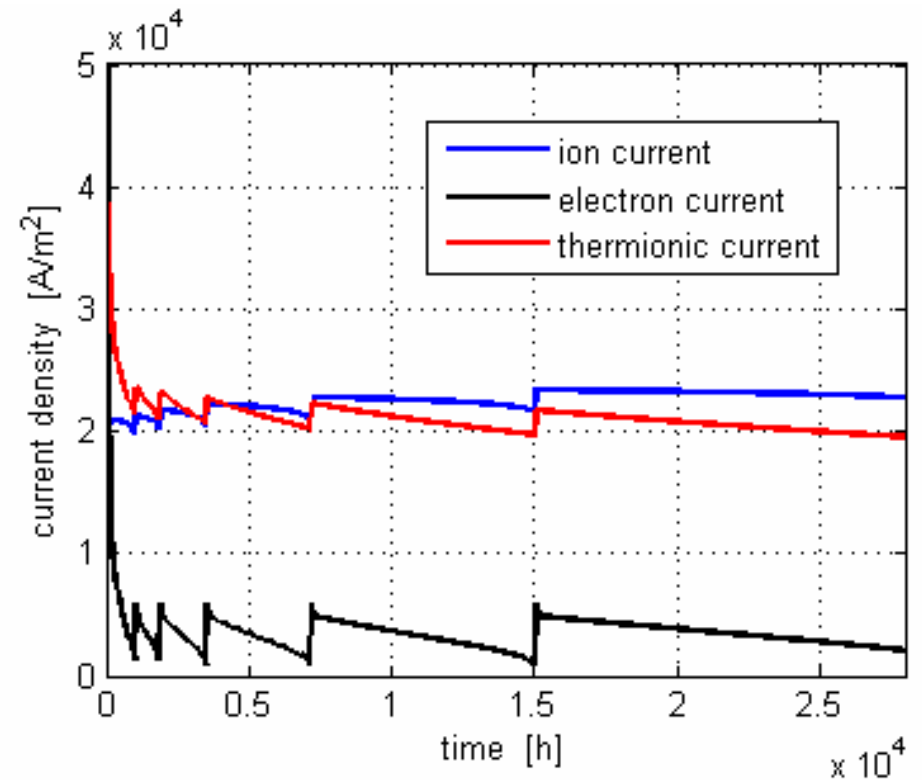

Fig. 11 Calculated currents to the cathode surface - 12A

As it can be noted also in Fig. 11, the currents plot shows the same step already noticed before. This is due to the fact the currents are calculated using the values reported in Fig. 10.

In none of the long duration cathode life tests an increase of the hollow cathode operating voltage (anode voltage) has been measured whereas in Fig. 10 the increase of the plasma voltage is clearly shown.

This can be explained noting that the anode voltage is the voltage "seen" by the power supply or, in other words, the voltage that the power supply need to supply to maintain the required discharge current.

This voltage is clearly a function of the plasma voltage, of the ionic, thermionic and electron current at the cathode surface and of the processes taking place in the orifice and in the

cathode plume.

$$
V_{\text {Pow Supply }}=V_{\text {surface }}+V_{\text {orifice }}+V_{\text {plume }}
$$

For what concerns the processes taking place at the cathode surface we can define the fraction of the total voltage relative to them as

$$
V_{\text {surface }}=\frac{1}{I_{D}} \int_{0}^{L_{c}}\left[J_{t h}(z)+J_{i}(z)-J_{e}(z)\right] \Delta V(z) 2 \pi r_{\text {int }} d z
$$

since the power provided by the power supply relatively to the cathode surface must be equal to the electrical power relative to the ion, electron and thermionic current flow at the surface.

Applying Eq. (16) we can calculate $V_{\text {surface }}$

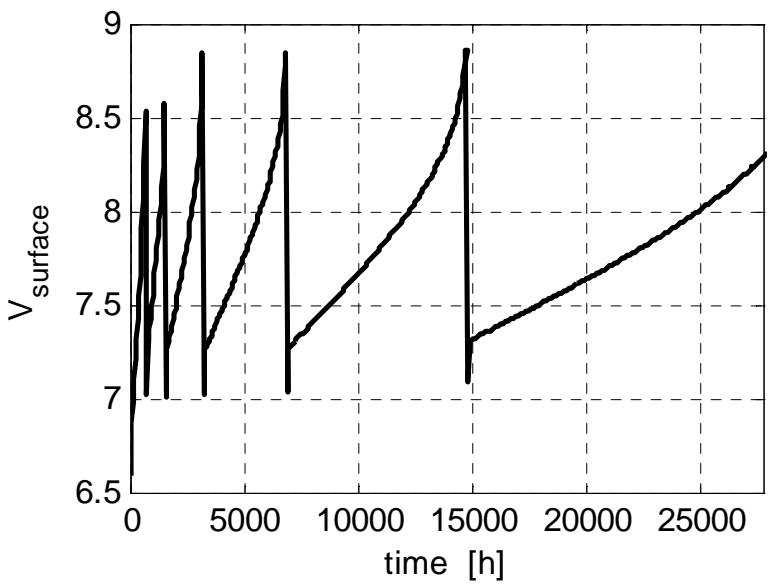

Fig. $12 \mathrm{~V}_{\text {surfac }}$ trend with time - 12A 
As it can be seen in the figure above despite of the wide variation in the plasma voltage the value of $V_{\text {surface }}$ oscillates around an average value that is more or less constant with time ( $7.7 \mathrm{~V})$ with variations of only $\pm 1 \mathrm{~V}$.

If this is compared to the experimentally recorded voltage (Fig. 13) we can see how this oscillation, even if generated by the assumptions made to simplify the plasma parameter update, has a value that is equal or smaller

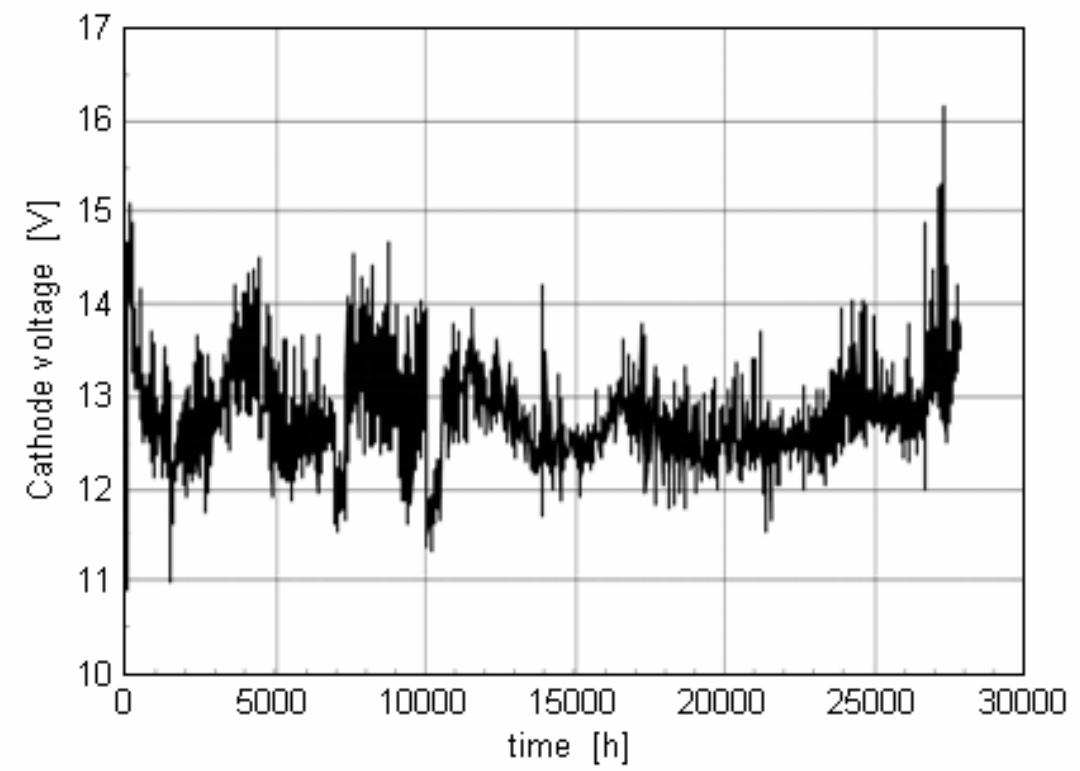

Fig. 13 Measured cathode voltage ${ }^{4}$ than the real oscillation of the voltage.

Noting what has been said above, noting that the average value of $V_{\text {surface }}$ is more or less constant with time and noting the comparison reported in Fig. 5 , we can conclude that the whole process used to update the plasma parameters gives reasonable results even when it is coupled with the surface coverage model.

From the data relative to the current densities we can calculate the value of the total current density and from it derive how the "active zone" of the cathode moves during life.

The total emission profiles along the insert

length are showed below for different times

As it can be seen at the beginning of life the total emission profile is smooth and gradually increasing from the upstream to the downstream end of the cathode. The "active zone" (the zone of highest emission) is then located at the downstream end.

As the cathode accumulates hours of operation the surface coverage changes due the depletion of low work function compounds starting from the downstream end of the insert. The active zone is then expected to move upstream.

Looking at Fig. 14 we can see how the emission profiles presents two distinct peaks of comparable intensity. The first peak at the downstream end of the cathode is due to the ion flux from the plasma to the insert surface and tends

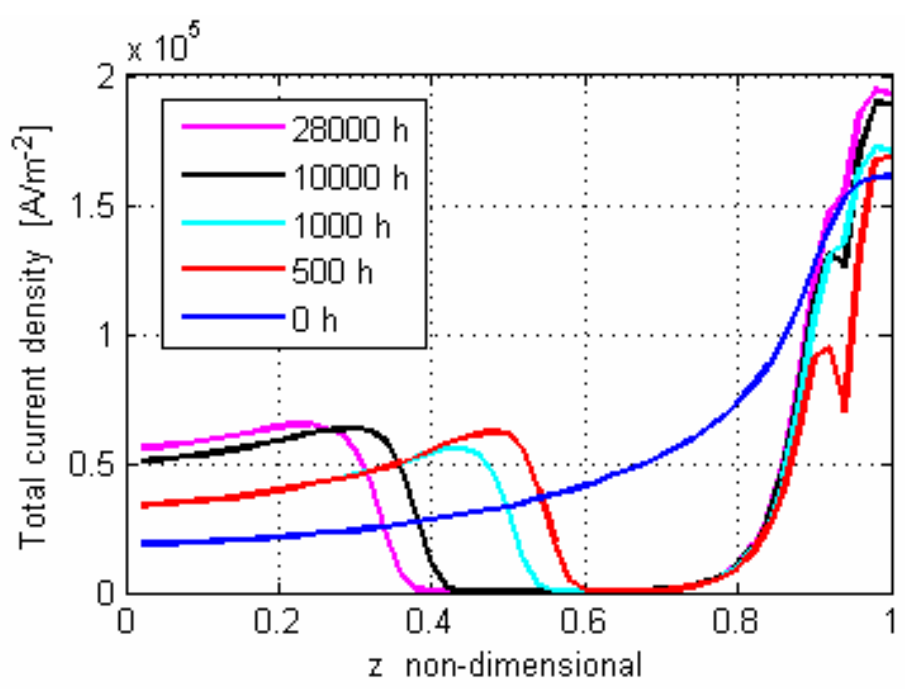

Fig. 14 Total emission profiles at different times - $12 \mathrm{~A}$ to increase with time to balance the reduction in thermionic emission due to low work function depletion. The second peak is located roughly at half the length of the cathode and is relative to the thermionic emission. This peak corresponds to the front of the low work function depletion and moves upstream with time following the depletion profiles shown in Fig. 7, 8. This peak is also decreasing in intensity as time goes on due to the fact that the moving upstream the insert temperature decreases consequently decreasing the thermionic emission. 
At the end of life the predicted emission zone is at $20 \%$ of the insert length from the upstream end of the cathode. Comparing this prevision with what has been derived by Sarver-Verhey in Ref. 4, 5 we can note how the model previsions are very conservative.

This can be explained noting the many conservative assumptions made in the development of this model like the neglection of the increase of temperature resistance of $\mathrm{Ba}_{3} \mathrm{WO}_{6}$ due to barium substitution by calcium, the assumption that the activation energy of the desorption process has the same value as the free Gibb's energy and the assumption that the barium evaporated from the insert will deposit only in the location close to where it has evaporated.

\section{End of life criterion development}

As shown in the paragraph above the surface coverage model and the plasma parameter updating processes produce results that are in qualitative and quantitative agreement with the expectations and with the measured data.

Once the evolution of the surface coverage has been derived we can now develop and end of life criterion.

Commonly in hollow cathodes operation the end of life is assumed to occur when the cathode cannot be started within the power supply capabilities.

This means that the cathode is sentenced to be dead when the ignition voltage goes beyond the maximum voltage that can be produced by the power supply.

A detailed modelling of the ignition process from first principle will require a full three-dimensional electrical transient simulation of the hollow cathode. In the literature the development of such a model has never been tried hence the problem will be solved in a semi-empirical way.

The voltage needed to cause the break down discharge in the cathode can be assumed to be inversely proportional to the electron density inside the cathode and in the cathode - keeper region.

Such electron density is certainly proportional to the emission from the cathode walls and hence from the thermionic emission from the insert.

$$
\Delta V_{\text {breakdown }} \propto \frac{1}{n_{e}} \propto \frac{1}{J_{t h}}
$$

The thermionic emission at the start-up is proportional to the surface coverage that can be achieved at the end of the start up process.

If we assume that the start up phase start at $t_{\text {start }}$ and lasts for $\Delta t_{\text {start }}$ seconds and that the insert is kept to a temperature $T_{\text {start }}$ during the start-up phase the total barium mass evaporated from the insert and the total mass needed to reach a full coverage are respectively

$$
\begin{aligned}
& m_{\text {BaO evap }}=\int_{0}^{\Delta t_{\text {start }}} \int_{S_{\text {insert }}} \dot{m}_{B a O}\left[T, \rho_{B a O}\left(t_{\text {start }}\right)\right] d S d t \\
& m_{\text {BaO needed }}=\left[\theta\left(t_{\text {start }}\right) S_{\text {insert }} \Pi+S_{O P}\right] \sigma_{B a O}
\end{aligned}
$$

If we assumed that the start up time is 10 minutes and that the temperature is $1100{ }^{\circ} \mathrm{C}$ the trend of $m_{B a O \text { evap }}$ and $m_{\mathrm{BaO}}$ needed with time is reported below. 


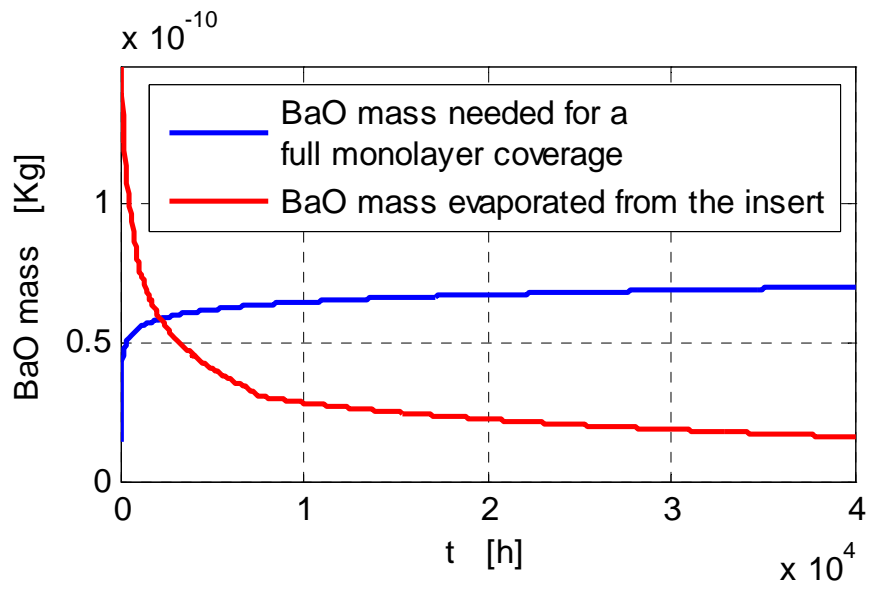

Fig. 15 BaO mass needed for a full coverage at startup and evaporated mass during startup Tstart $=1100{ }^{\circ} \mathrm{C}-12 \mathrm{~A}$
Once the needed and deposited mass are know the average coverage can be calculated as

$$
\theta_{\text {startup }}=\theta\left(t_{\text {start }}\right)+f a \frac{m_{\text {BaO evap }}}{m_{\text {BaO needed }}}
$$

From this value of the coverage the average work function can be calculated and so the thermionically emitted current.

Since from Eq. (17) we only know that the voltage is proportional to $1 / J_{t h}$ we can define a voltage breakdown amplification factor as

$$
\beta=\frac{J_{t h 0}}{J_{t h}(\theta)}
$$

where $J_{t h 0}$ is the emission current relative to the temperature $T_{\text {start }}$ and to a full surface low work function coverage. The calculated trend of the amplification factor for the cathode tested by SaverVerhey ${ }^{4,5}$ is reported in Fig. 16.

The experimental value of the amplification factor can be calculated from the data published by Server-Verhey ${ }^{4,5}$ and is reported in Fig. 17.

Comparing the data in Fig. 16 and Fig. 17 it can be seen how both the time values and the amplification values are out of scale. In spite of this the shape of the experimental and numerical $\beta$ curves are quite close showing a phase were the start up voltage stays constant (numerical 0-2,000 hours / experimental 0-21,000 hours) a phase of quick increase of the voltage (numerical 2,000-8,000 hours / experimental 22,000-24,000 hours) and then a phase of slower constant rate increase (numerical from 8,000 hours onwards / experimental 24,000 hours onwards).

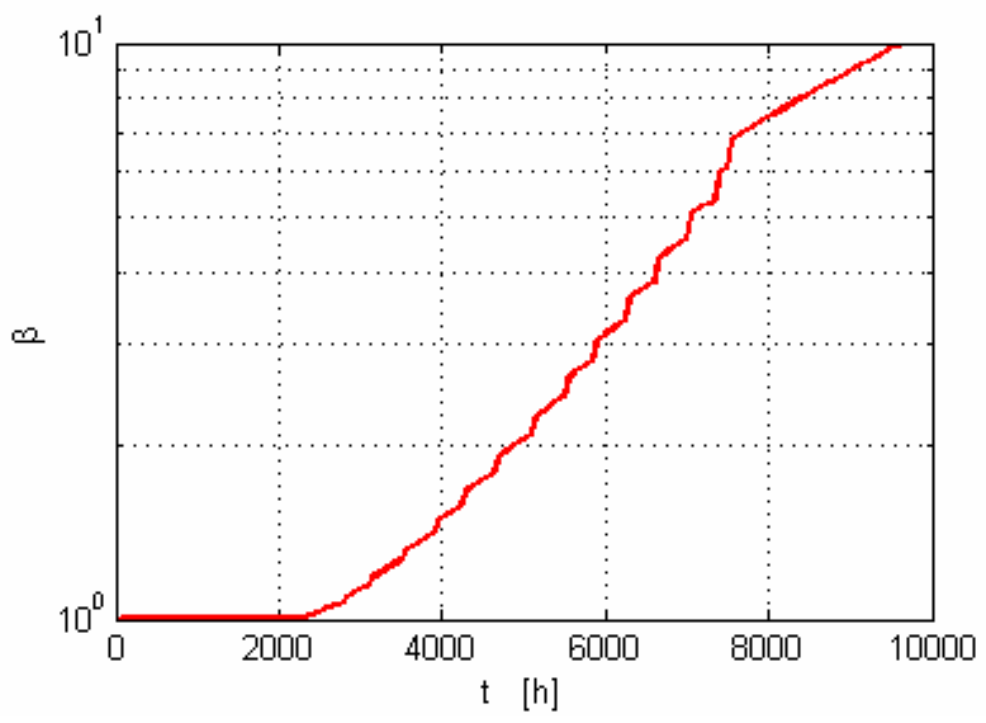

Fig. 16 Calculated voltage amplification factor - 12A 


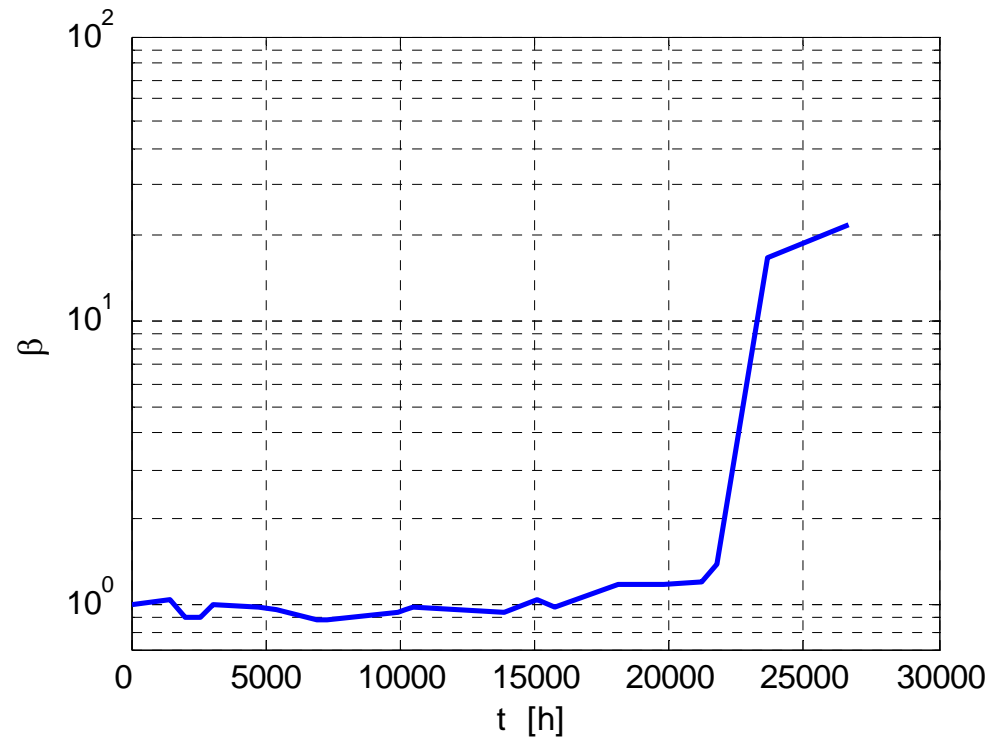

Fig. 17 Experimental voltage amplification factor ${ }^{4}$
The shift in the timescale can be explained noting that in the model a lot of conservative hypotheses have been done resulting in a voltage increase (cathode death) that for $1100{ }^{\circ} \mathrm{C}$ happens much before than in reality. The difference in the amplification values can be explained noting that Eq. (17) represents only a very easy interpretation of the relation between surface coverage and start up voltage.

The qualitative similarities found between these two trends give us an evidence of the goodness of the model stressing its conservative nature and give us also a clear end of life criterion.

Comparing Fig. 15 and Fig. 16 we can note how the voltage increase happens when the evaporated barium oxide mass becomes less than the needed one

hence and end of life criterion can be state as:

"Given the temperature and the duration of the start up procedure the end of life of a cathode is reached when the barium oxide mass evaporated from the insert during the start up phase is not enough to provide a full monolayer coverage over all the internal cathode surfaces"

Using this criterion we will now change $T_{\text {start }}$ until the end of life of the cathode reported in Ref 4 matches the experimental value of 28,000 hours.

The experimental value is matched when the start up temperature is $1150{ }^{\circ} \mathrm{C}$, the relative graph of the evaporated and needed mass is reported below

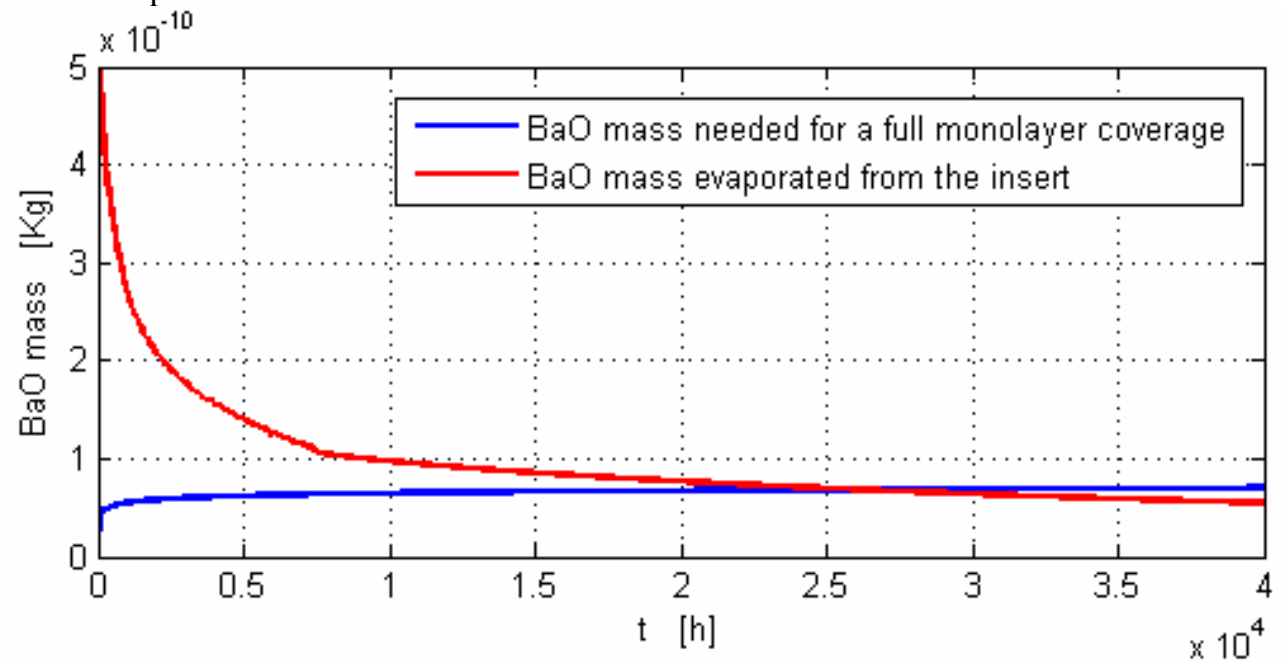

Fig. $18 \mathrm{BaO}$ mass needed for a full coverage at startup and evaporated mass during startup $\mathrm{T}=1150{ }^{\circ} \mathrm{C}-$ 12A

Now that an end of life criterion has been developed and tuned with the data in Ref 4 the ELT NSTAR discharge cathode will be simulated. 


\section{ELT NSTAR cathode surface coverage and lifetime simulation}

The ELT discharge cathode has been simulated for the throttle levels TH0, TH8 and TH15 and then it has been simulated using the throttle level history relative to the Deep Space $1 \mathrm{flight}$ spare ion engine test reported in Table 3.

\begin{tabular}{ccc}
\hline \hline \multicolumn{2}{c}{ Table 3} & - ELT discharge cathode throttle settings ${ }^{\mathbf{3}}$ \\
\hline \hline \multirow{2}{*}{ TH level } & Accumulated hours & Discharge Current \\
\hline 12 & 500 & 9.9 \\
15 & 4800 & 13.5 \\
8 & 10500 & 7.6 \\
15 & 15500 & 13.5 \\
0 & 21500 & 4.9 \\
15 & 25500 & 13.5 \\
5 & 30000 & 6.9 \\
\hline \hline
\end{tabular}

Below all the data relative to surface coverage, work function, and end of life will be reported for every throttle setting.

A. THO - $4.9 \mathrm{~A}$

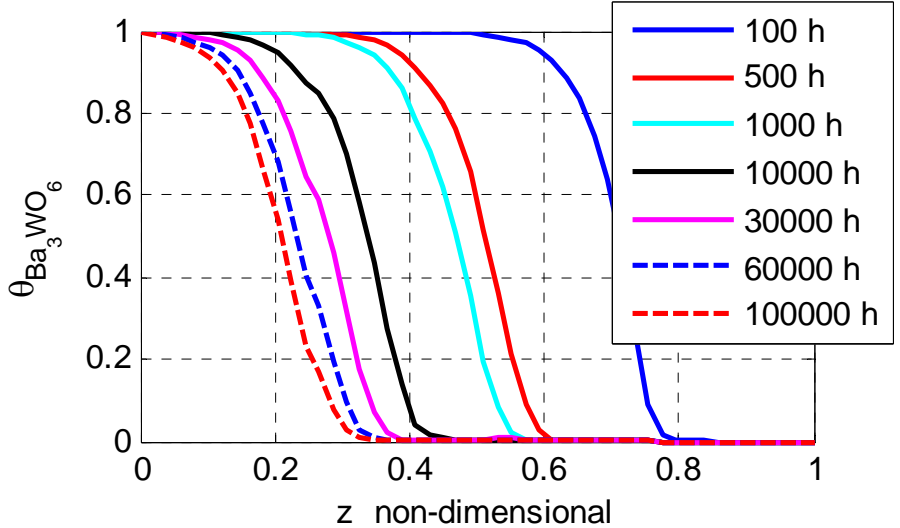

Fig. $19 \mathrm{Ba}_{3} \mathrm{WO}_{6}$ surface coverage - TH0

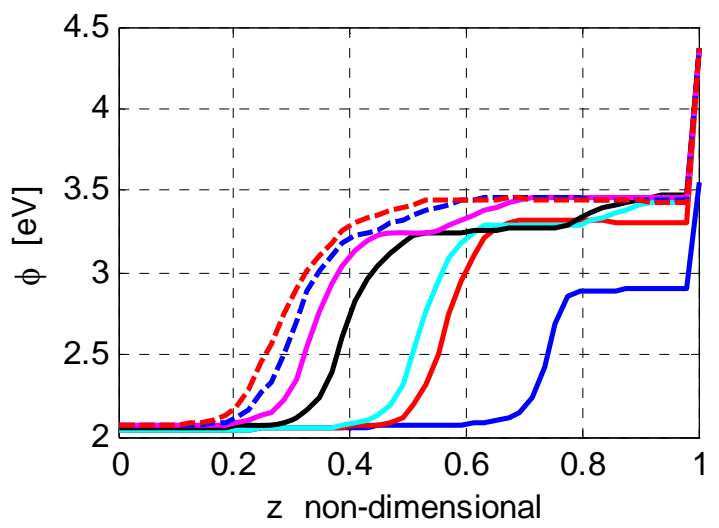

Fig. 21 surface work function - THO

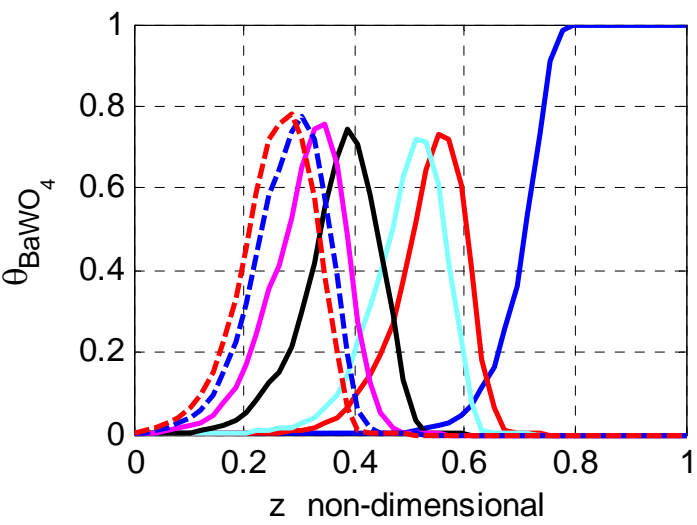

Fig. 20 BaWO $_{4}$ surface coverage - THO

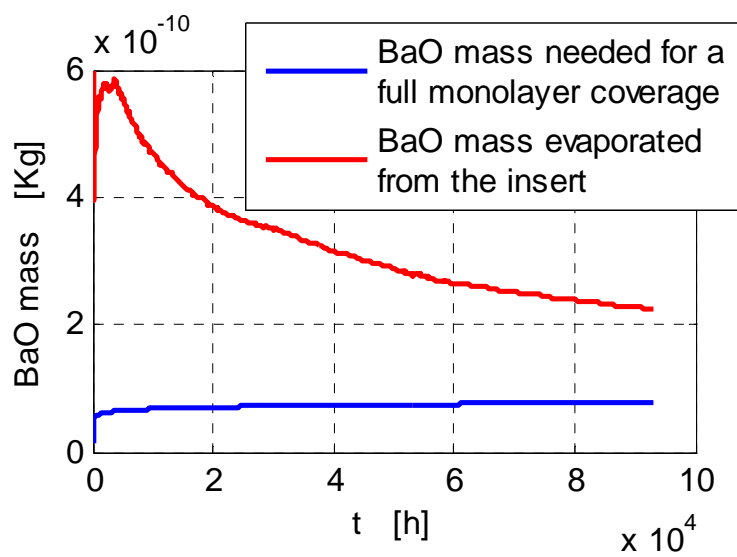

Fig. 22 BaO mass needed for a full coverage at startup and evaporated mass during startup $\mathrm{T}=1150$ ${ }^{\circ} \mathrm{C}-$ THO 
All the comments made about the cathode tested in Ref 4 relatively to the low work function coverage and the work function evolution are still valid for the ELT cathode. Regarding the end of life a 100 thousands hours simulation shows that the cathode is still far from the end of life. From an interpolation of the curves in Fig. 22 the end of life can be estimated to occur after 200,000 hours.

B. TH8 - 7.6A

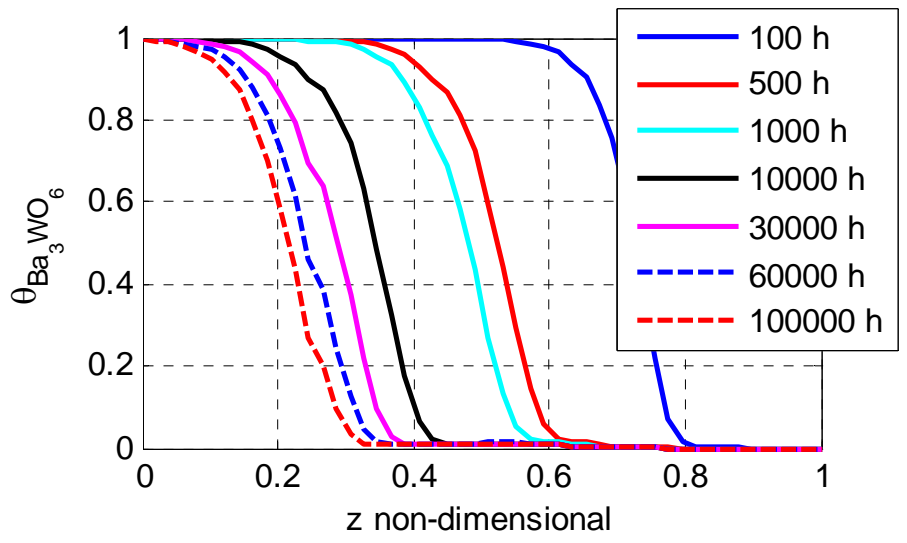

Fig. $23 \quad \mathrm{Ba}_{3} \mathrm{WO}_{6}$ surface coverage - TH8

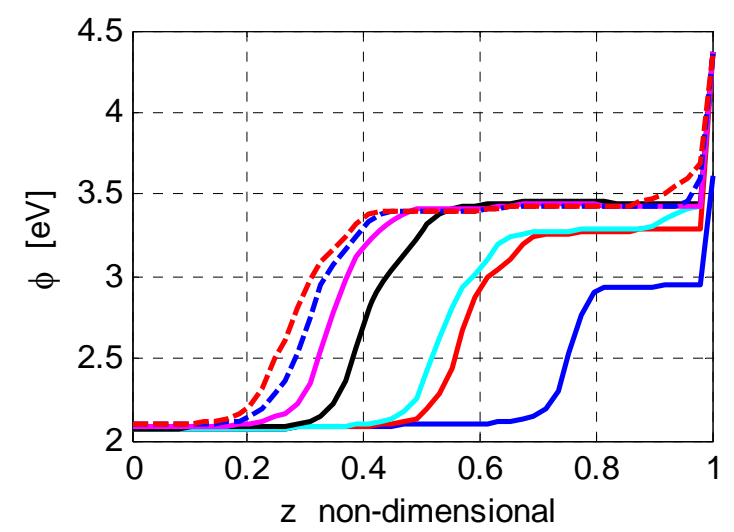

Fig. 25 surface work function - TH8

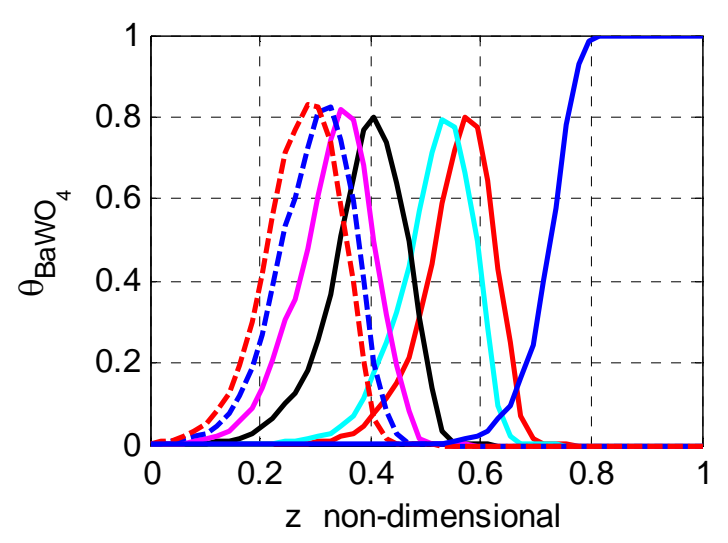

Fig. $24 \mathrm{BaWO}_{4}$ surface coverage - TH8

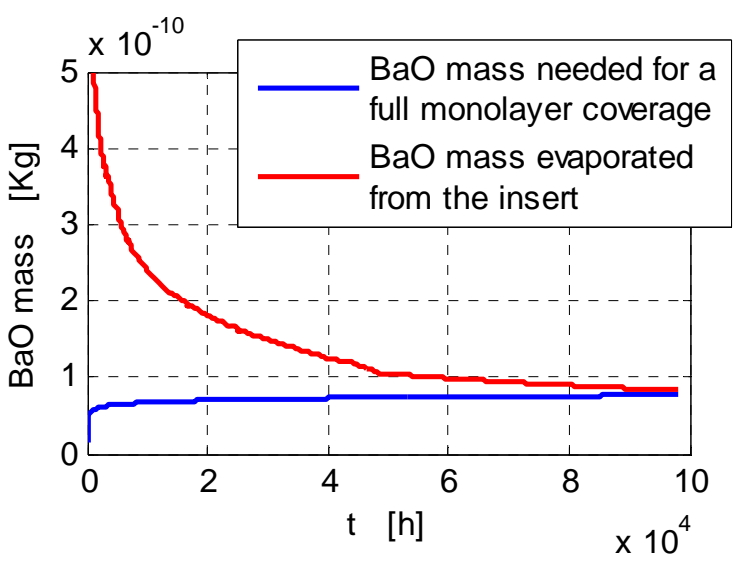

Fig. 26 BaO mass needed for a full coverage at startup and evaporated mass during startup $\mathrm{T}=1150{ }^{\circ} \mathrm{C}-\mathrm{TH8}$

The surface coverage and work function evolution are similar to the one reported for TH0 whereas the lifetime is shown to be 100,000 hours. The strong reduction in lifetime is not due to a higher low work function depletion (since the work function profiles in Fig. 21 and Fig. 25 and are quite similar and also the needed mass reported in Fig. 22 and Fig. 26 have similar values) but to a quicker ageing of the insert chemistry due to the fact that the insert temperature is higher at TH8 than at TH0.

C. TH15 - 13.5A 


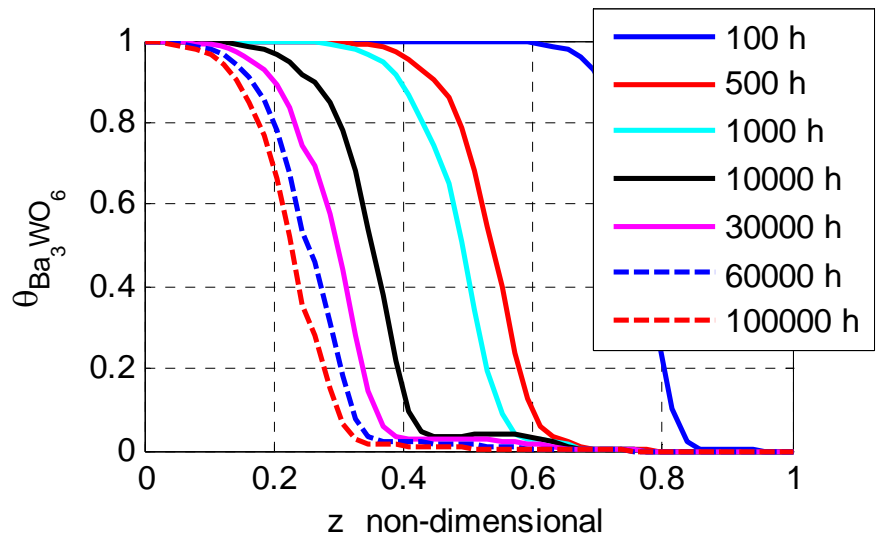

Fig. $27 \mathrm{Ba}_{3} \mathrm{WO}_{6}$ surface coverage - TH15

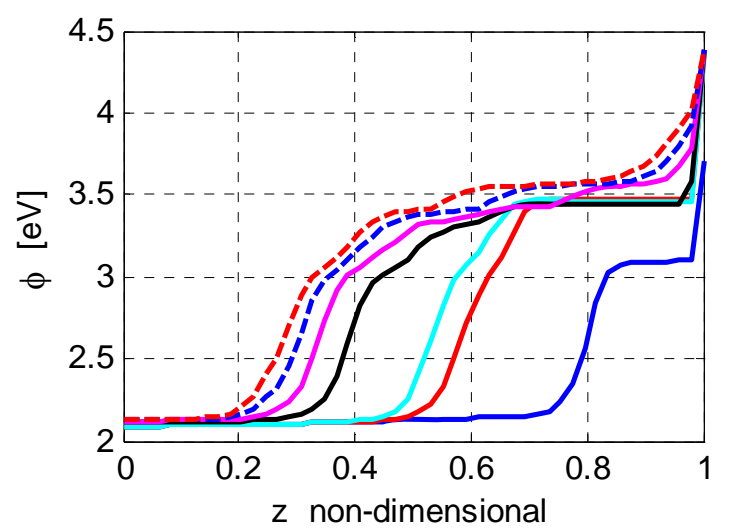

Fig. 29 surface work function - TH15

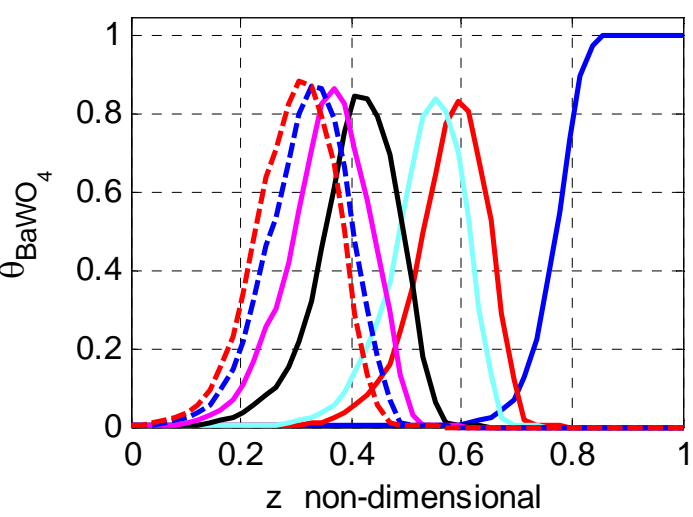

Fig. $28 \mathrm{BaWO}_{4}$ surface coverage - TH15

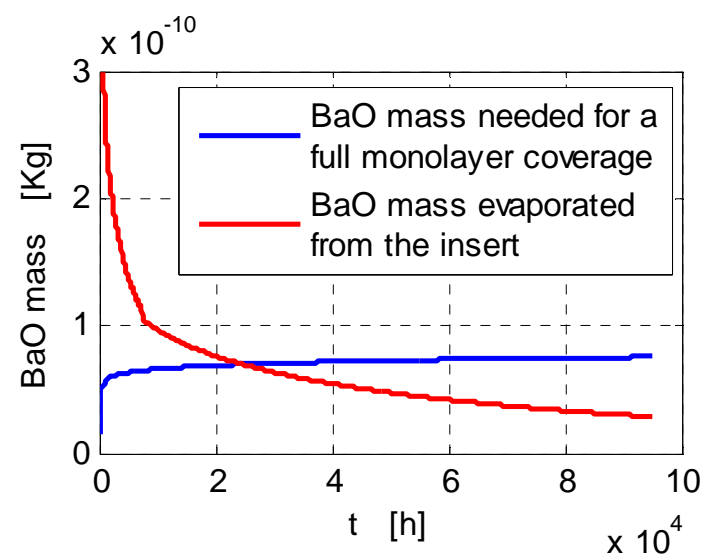

Fig. 30 BaO mass needed for a full coverage at startup and evaporated mass during startup $\mathbf{T}=1150{ }^{\circ} \mathbf{C}-\mathbf{T H 1 5}$

For TH15 the low work function coverage and $\phi$ profiles are still similar to the ones relative TH8 and TH0 although the relatively high insert temperature causes a quicker aging of the insert chemistry making the evaporated barium oxide mass drop below the needed mass after about 25,000 hours hence fixing to this value the life of the cathode. Such a low value could be expected since the operating conditions of the TH15 throttle level are close to the ones used in the lifetest reported in Ref 4 where the lifetime was 28,000 hours.

The similarities of the low work function coverage profile between this three throttle levels can be explained noting that even if at lower TH we have higher voltage drops and hence higher energy of the sputtering ions (Fig. 5, 6 ) the lower average insert temperature results in an higher activation energy ${ }^{18}$, hence counterbalancing the increase in the sputtering energy; it must also be noted that a lower insert temperature means also a slower aging of the chemistry that on a very long time scale means higher local barium oxide concentrations and hence higher evaporation rates hence helping to balance the increase in the sputtering energy.

Regarding the hollow cathode lifetime a simple "rule of thumb" exists according to which the dispenser life doubled for every $30^{\circ}$ to $40^{\circ}$ reduction in the operating temperature of the insert. This rule has been derived from the analyses of a large volume of data regarding the life of cathodes dispensers for travelling wave tubes and CRT devices and is reported in Ref. 13, 14. 17.

In TWT and CRT devices there is no contact between the plasma and the insert surface hence no ion bombardment occurs, changing the depletion scenario with respect to a hollow cathode for space application.

This rule has never been tested with hollow cathodes for ion thrusters hence it will be tested with the data obtained numerically with the model.

The predicted lifetimes and the average temperatures relative to TH0, TH8 and TH15 are reported below 
Table 4 - Lifetime and average insert temperatures for different THs

\begin{tabular}{ccccc}
\hline \hline TH level & $\begin{array}{c}\text { Average insert } \\
\text { temperature }\left[{ }^{\circ} \mathrm{C}\right]\end{array}$ & $\begin{array}{c}\text { Predicted } \\
\text { lifetime [h] }\end{array}$ & $\Delta_{\text {Life }}$ & $\Delta T$ \\
\hline 0 & 1045 & 200,000 & 1 & 0 \\
8 & 1104 & 100,000 & $1 / 2$ & 59 \\
15 & 1169 & 25,000 & $1 / 8$ & 124 \\
\hline \hline
\end{tabular}

To verify the statement reported above relatively to the lifetime trend with temperature we will now interpolate the data in Table 4 normalizing them relatively to THO and using an interpolating function of the kind

$$
\Delta_{\text {Life }}=2^{-\frac{\Delta T}{b}}
$$

where $\Delta_{\text {Life }}$ is the lifetime amplification factor.

A mean least squares optimization returned a value of $b=51^{\circ}$ that, as it can be seen in Fig. 31, produce a fit that well interpolates the data hence showing how the prediction of the model are in qualitative agreement with the expectation based on the analysis of a large amount of data relative to dispensers in TWTs and CRTs showing how

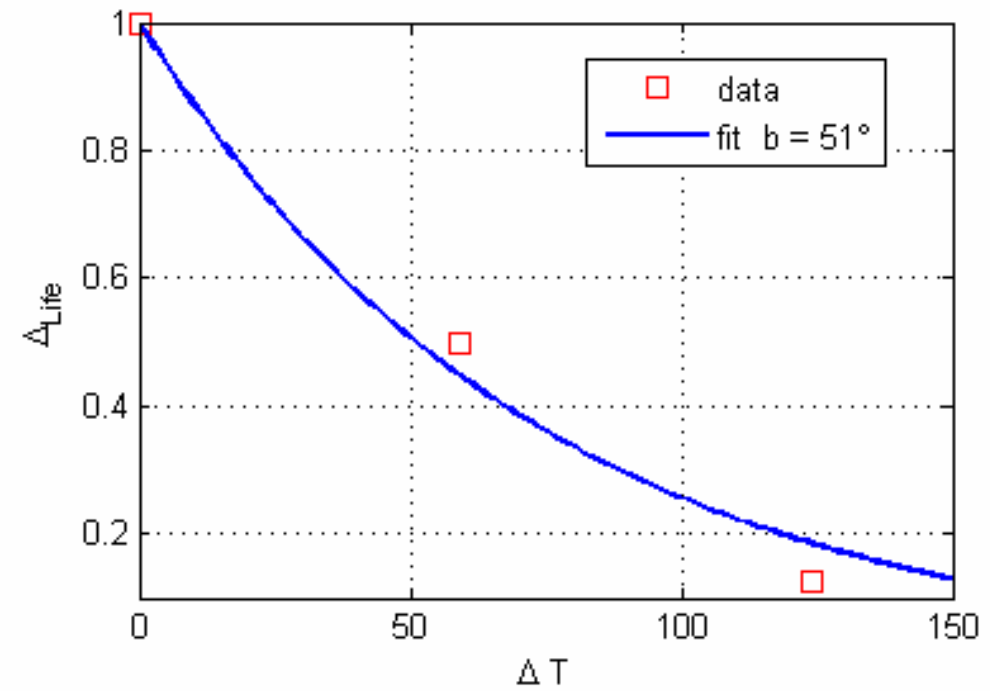

Fig. 31 Lifetime trend with temperature, computed data and interpolation the life time double every 51 degrees of temperature decrease.

As said before this rule of thumb is based on observation made on TWT and CRT devices and hence does not take into account the effects of ion bombardment, whereas it should reflect the effects of thermal desorption and deposition.

In this particular case the rule seems to be applicable (we can not make any definitive statement since only 3 point have been derived).

This might be explained noting, as already said before, how, in this particular case, the surface coverage profiles are very similar for all the throttle levels given the counteracting effects due to the insert temperature decrease and to the sheath voltage fall increase.

Since the surface coverage profiles are similar for all the THs

the evaporation rate of barium oxide from the insert is the critical parameter. The behaviour of such evaporation is probably similar in hollow cathodes and in TWT and CRT devices hence allowing the application of the "rule of thumb” reported above.

This rule is instead most probably not applicable between different cathodes since the behaviour of the voltage drop and of the insert temperature profiles will be different, and will not be applicable in cases where a variation of the discharge current produces variation of voltage and of the temperature that are too big or too small to reciprocally balance.

Now that the ELT cathode has been simulated using at TH15, TH8 and TH0 we can move to its simulation using the throttle profile used in Ref 3.

\section{Discharge cathode from the Deep Space 1 spare ion engine 30,000 hour life test}




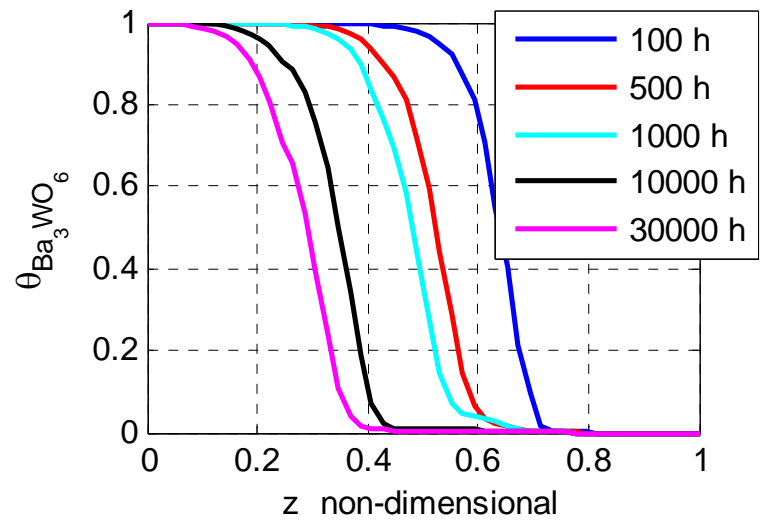

Fig. $32 \mathrm{Ba}_{3} \mathrm{WO}_{6}$ surface coverage - NSTAR

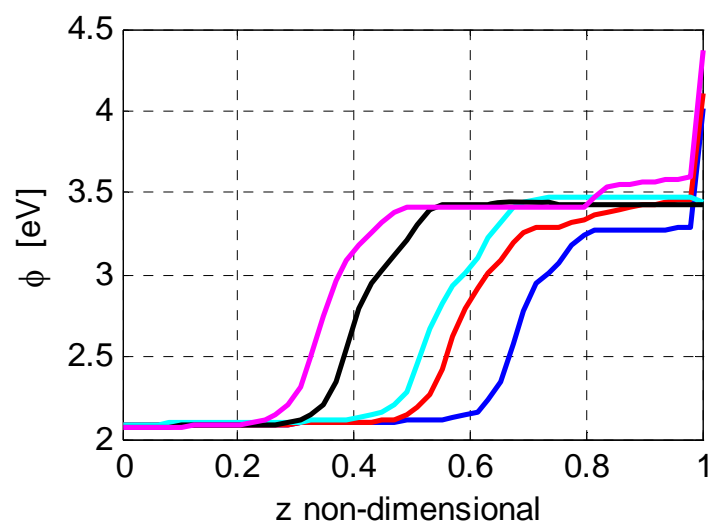

Fig. 34 surface work function - NSTAR

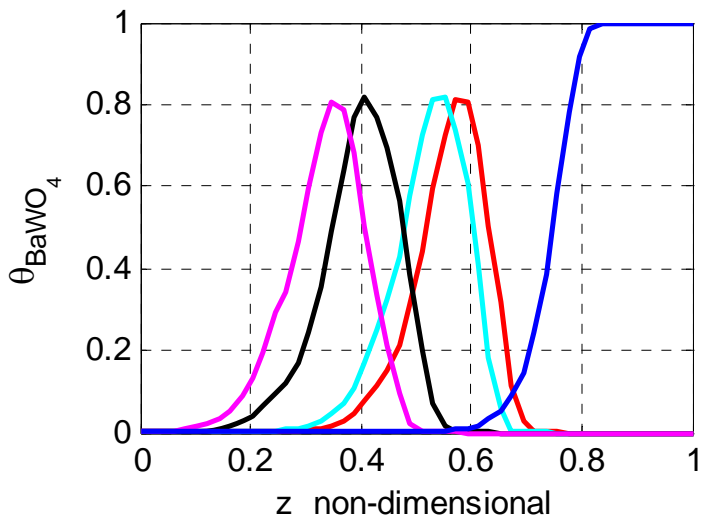

Fig. $33 \mathrm{BaWO}_{4}$ surface coverage - NSTAR

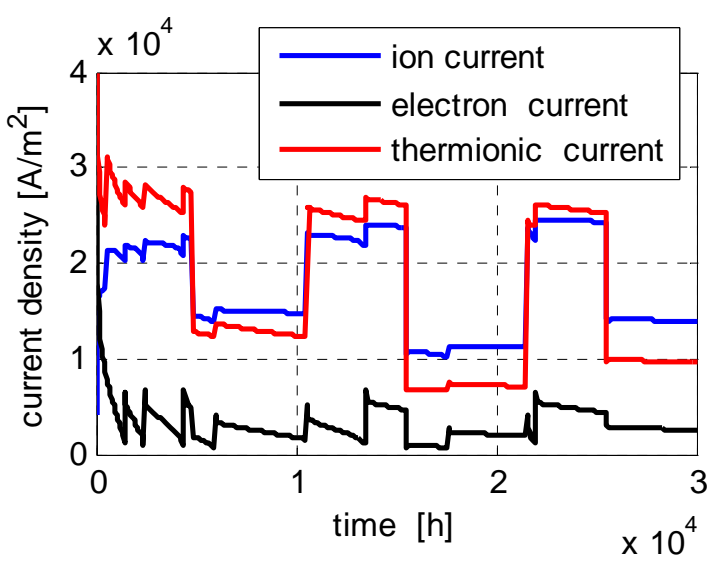

Fig. 35 current densities to the cathode surface NSTAR

Regarding coverage and work function always the same comments are valid. For the currents plot we can note how when the cathode is run at TH15 the emitted current is higher than the ion one while at all the other THs we have the contrary. This can be explained noting that at TH15 the ion bombardment energy is lower due to the lower sheath voltage producing a lower desorption rate and that TH15 is the TH level with the highest insert temperature

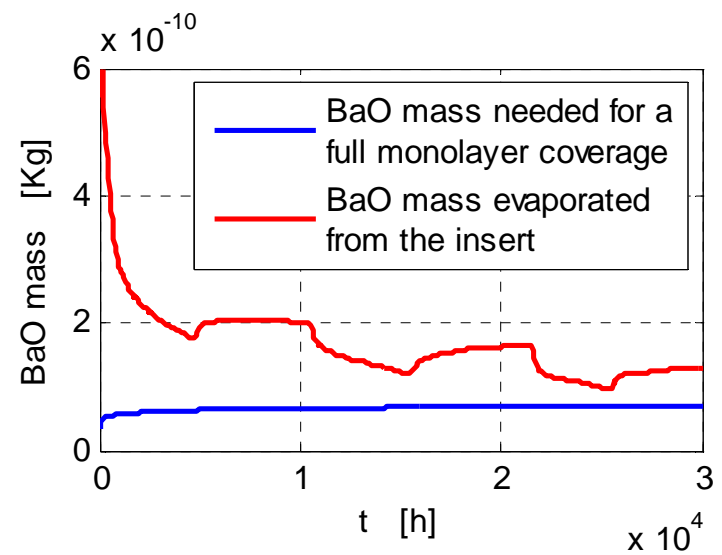

Fig. 36 BaO mass needed for a full coverage at startup and evaporated mass during startup $\mathrm{T}=1150{ }^{\circ} \mathrm{C}-\mathrm{NSTAR}$ hence with the highest thermionic emission.

Looking at Fig. 36 we can note how after 30,000 hours the cathode has still not reached the end of life. This is in accordance with the real test of the cathode that has been voluntary stopped after 30,000 hours.

The estimation of the lifetime of the cathode of course depends on the throttle level the cathode will be run at from 30,000 hours on.

As it can be seen in Fig. 36 during the time when the cathode is run at TH15 we have a net reduction of the evaporated mass while during the other TH levels we have even an increase in the evaporated mass.

This increase can be explained noting that the evaporation is proportional not only to the temperature but also on the local $\mathrm{BaO}$ concentration as demonstrated in Ref. 15.

When the cathode moves from TH15 to a lower throttle level the insert temperature will decrease. A lower temperature means a lower evaporation rate hence a higher possibility that by diffusion the $\mathrm{BaO}$ depletion at the surface can be replenished. 
This at the beginning will result in a local increase in the $\mathrm{BaO}$ concentration that will lead to an higher evaporation rate explaining while at the beginning of TH8 $(4,800 \mathrm{~h}), \mathrm{TH} 5(15,500 \mathrm{~h})$ and TH0 $(21,000 \mathrm{~h})$ the total deposited $\mathrm{BaO}$ mass increase with time.

After some time this higher evaporation rate will bring the system to an equilibrium between diffusion and evaporation (TH8 7,500 h) and then to a gradual reduction of the surface $\mathrm{BaO}$ density with a subsequent decrease in the evaporation rate (TH8 7,500-10,000 h).

It can be useful to estimate the lifetime of the cathode assuming that the operating conditions from 30,000 hours on will reflect those used until 30,000 hours. To do so the trends in Fig. 36 have been extrapolated up to 100 thousands hours.

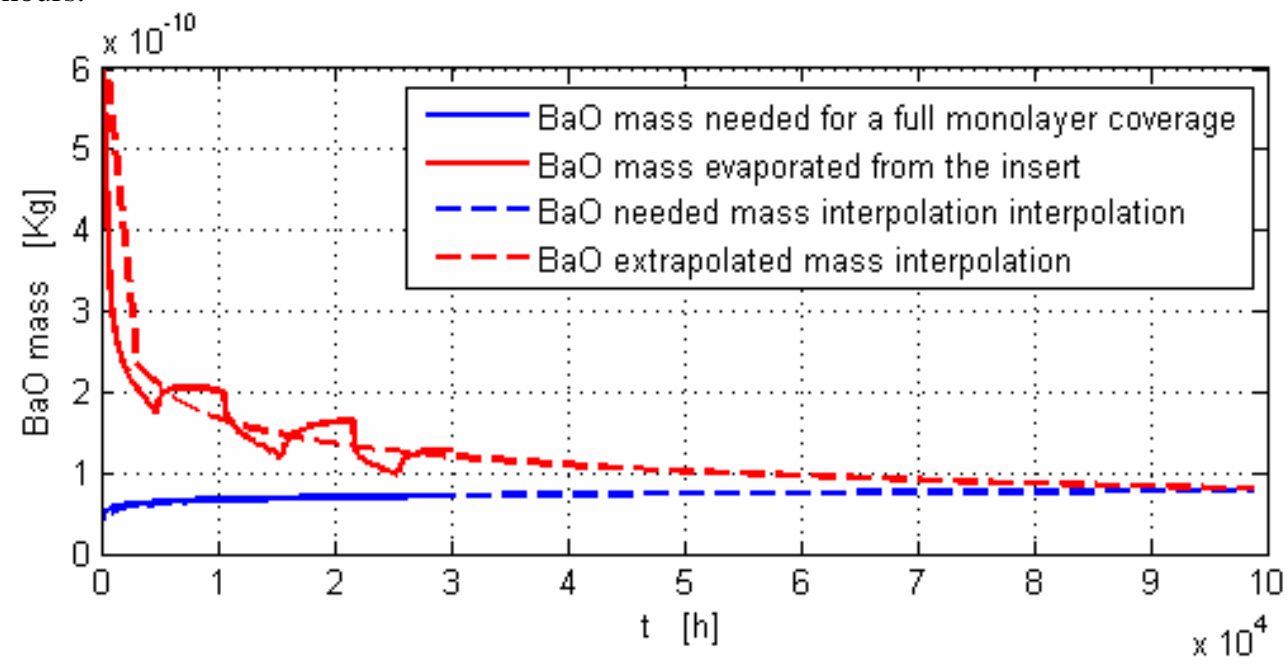

Fig. 37 extrapolation of the deposited and needed $\mathrm{BaO}$ mass $\mathrm{T}=1150$ - NSTAR

From this extrapolation a life time of 100 thousand hours can be predicted. The lifetime so predicted might seem too long considering that the lifetime at TH15 is only 25,000 hours. This can be explained calculating the average discharge current of the cathode using the nominal current of each $\mathrm{TH}$ level weighted with the time that each $\mathrm{TH}$ level has been used for. The value so obtained is $7.98 \mathrm{~A}$ very close to the one of TH8 that is $7.6 \mathrm{~A}$, in fact the lifetime predicted from Fig. 37 is very close to the one relative to TH8.

The lifetime of 100,000 hours is then valid if the cathode is run at TH8 from 30,000 hours onward or if the throttle history reported in Table 3 is repeated for other 70,000 hours keeping the same ratio between the various throttle levels but using smaller time intervals so that the overall trend will get closer to the average one.

If the cathode is run at TH15 the lifetime is going to be around 35,000 hours whereas if the throttle level used is lower than TH8 the lifetime will be in excess of 100,000 hours.

\section{Conclusions}

In this paper the deposition and desorption model already developed by the authors has been improved and coupled with a semi-empirical plasma parameter update procedure.

The process used to update the plasma characteristic has been validated using the data available in the literature finding a very good agreement.

The cathode from Ref 4, 5 and the one from the Deep Space 1 spare ion engine 30000 hours test $^{3}$ have been then simulated.

The simulation of the cathode in Ref 4, 5 have shown how the low work function compounds coverage tends to decrease with time and how this decrease is followed by an increase in the plasma voltage. The effect of this increase has been analyzed and the reason why the predicted increase in the plasma voltage does not correspond to an increase of the voltage supplied by the power supply has been explained noting how the change in the current density distribution on the surface balance the voltage increase so that the current weighted mean voltage remains constant.

The evolution of the active area of the cathode has been studied. It has been shown that the downstream of the cathode is always a high current density area thanks to the high flux of ions reducing at this location of the surface whereas the peak due to thermionic emission tends to move upstream with time. 
From the data obtained with the simulation of the cathode in Ref. 4 an end of life criterion has been derived and the model has been tuned to predict a lifetime of 28,000 hours fitting the experimental evidence for this cathode.

This end of life criterion has then been applied to the NASA ELT discharge cathode relatively to the throttle levels TH0, TH8, TH15 and to the throttle profile used in the experimental analysis ${ }^{3}$.

The results obtained show a lifetime of about 200,000 hours for TH0, 100,000 for TH8 and 25,000 for TH15. These data have been interpolated trying to verify the rule of thumb reported in Ref. 13, 14 finding a good agreement. The limit in the applicability of this rule has also being investigated and the cases where this can be used have been defined.

The simulation of the cathode with the throttle profile used during the experimental life test shows how the cathode life time excess 30,000 hours as observed in the experimental test reported in Ref 3 .

The lifetime of the cathode is then relative to the throttle profile that will be used from 30,000 hours onwards, if TH8 (equivalent to the mean discharge current used up to 30,000 hours) is used the predicted lifetime is 100,000 hours whereas higher throttle level will provide shorter lifetimes (TH15 will provide a lifetime of about 35-40 thousand hours) and lower throttle levels longer lifetime (TH0 150-200 thousand hours).

Considering what said above we can conclude that the model presented in this paper gives results that are in agreement with the experimental evidence and with the theoretical expectations resulting to be, at present, together with the barium depletion model ${ }^{15}$ and with the deposition and desorption model ${ }^{17}$ one of the most comprehensive hollow cathode life-time model and the only one that, currently, includes a form of coupling between the surface changes and the plasma characteristics and that takes into account (even if indirectly through the results of the barium depletion chemical model) the complex chemistry of the impregnant.

\section{References}

${ }^{1}$ M. Coletti, A. Grubisic, N. Wallace, "European Student Moon Orbiter Solar Electric Propulsion Subsystem Architecture. An All - Electric Spacecraft”, IEPC-2007-111, 30th Electric International Propulsion Conference, Florence, Italy, September 2007.

${ }^{2}$ M. Coletti, A. Grubisic, C. Collingwood, N. Wallace, N. Wells, S.B. Gabriel, "European Student Moon Orbiter Solar Electric Propulsion Subsystem Architecture. An All - Electric Spacecraft”, Acta Astronautica (to be published)

${ }^{3}$ Sengputa, A. "Destructive Physical Analysis of Hollow Cathodes from the Deep Space 1 Flight Spare Ion Engine 30,000 $\mathrm{Hr}$ Life Test”. 29th International Electric Propulsion Conference, IEPC-2005-026, 2005.

${ }^{4}$ T. R. Sarver-Verhey, "Destructive Evaluation of a Xenon Hollow Cathode After a 28,000 Hour Life Test", NASA/CR1998-208678, 1998

${ }^{5}$ T. R. Sarver-Verhey, “Scenario for Hollow Cathode End-of-Life”, NASA/CR—2000-209420, 2000

${ }^{6}$ I. Mikellides, D. Katz, D. Goebel and J. Polk, ”Model of Hollow Cathode Insert Plasma”, AIAA-2004-3817, 40th AIAA/ASME/SAE/ASEE Joint Propulsion Conference and Exhibit, Fort Lauderdale, Florida, July 11-14, 2004

${ }^{7}$ I.G. Mikellides , I. Katz, D.M. Goebel, J.E. Polk, "Hollow Cathode Theory and Experiment. A Two-Dimensional Model of the Emitter Region”, J. of App. Physics, vol. 98, 2005

${ }^{8}$ I Katz, J. E. Polk, I. G. Mikellides et al. , “Combined Plasma and Thermal Hollow Cathode Insert Model”, IEPC-2005-228, 2005, 29th Electric International Propulsion Conference ,Princeton, USA, October 2005.

${ }^{9}$ I.G. Mikellides , I. Katz, D.M. Goebel, J.E. Polk, "Theroretical Model of a Hollow Cathode Plasma for the Assesment of Insert and Keeper Lifetimes”, 41 $1^{\text {st }}$ AIAA Joint Propulsion Conference, Arizona, 2005

${ }^{10}$ J. Polk, A. Grubisic, N. Taheri, D. Goebel, R. Downey, S. Hornbeck, "Emitter Temperature Distributions in the NSTAR Discharge Hollow Cathode”, AIAA-2005-4398

${ }^{11}$ J.M. Roquais, F. Poret, R. le Doze et al., "Barium Depletion Study on Impregnated Cathodes and Lifetime Prediction", Applied Surface Science 215, 2003, pp 5-17

${ }^{12}$ R.T. Longo, "Physics of Thermionic Dispenser Cathode Aging”, Journal of Applied Physics, Vol 94, No 10, 2003

${ }^{13}$ I. Katz, et al. "Model of Hollow Cathode Operation and Life Limiting Mechanisms". IEPC-2003-0243, 2003.

${ }^{14}$ Goebel, D.M. et al. "Extending Hollow Cathode Life for Electric Propulsion in Long Term Missions”. AIAA-2004-5911, Space 2004 Conf. and Exhibit, 2004.

${ }^{15}$ M. Coletti, S.B. Gabriel, “A Chemical Model for Barium Oxide Depletion from Hollow Cathode's Insert”, AIAA-20075193, 43rd AIAA/ASME/SAE/ASEE Joint Propulsion Conference \& Exhibit, Cincinnati, Ohio, USA, July 2007.

${ }^{16}$ M. Coletti, A. Grubisic, S.B. Gabriel, "Numerical Simulation of the Insert Chemistry of the Hollow Cathodes from the Deep Space 1 Ion Engine 30,000 Hrs Life Test”, IEPC-2007-113, 30th Electric International Propulsion Conference, Florence, Italy, September 2007.

${ }^{17}$ I.M. Ahmed Rudwan, N. Wallace, M. Coletti, S.B. Gabriel, "Emitter Depletion measurement and modelling in the T5\&T6 Kaufman-type Ion Thrusters”, IEPC-2007-256, 30th Electric International Propulsion Conference, Florence, Italy, September 2007.

${ }^{18}$ M. Coletti, S.B. Gabriel, “A Model for Low Work Function Compound Deposition on Hollow Cathode Insert Surface”, 46th AIAA Aerospace Sciences Meeting and Exhibit, Reno, Nevada, USA, January 2008 
${ }^{19}$ M. Coletti, S.B. Gabriel, "Hollow Cathode Modelling”, 5th International Spacecraft Propulsion Conference, Heraklion, Crete, Greece, May 2008

${ }^{20}$ T.N. Resulhina, V.A. Levitskii, M.Ya. Frenkel, Izvestiya Akademii Nauk SSSR, Neorgan. Mater. 2, 1966, pp. 325-331.

${ }^{21}$ L.L.Y. Chang, M.G. Scroger, B. Phillips, "Alkaline-Earth Tungstates: Equilibrium and Stability in the M - W - O Systems”, Journal of the American Ceramic Society, Vol 49, n 7, 1966, pp 385-390

${ }^{22}$ B. Phillips, L.L.Y. Chang, "High Temperature Stability of Tungsten Oxide Structures", Transaction of The Metallurgic Society of AIME, Vol 230, 1964, pp 1203-1206.

${ }^{23}$ E.R. Kreidler, "Phase Equilibria in the System $\mathrm{CaO}-\mathrm{BaO}-\mathrm{WO}_{3}$ ", Journal of the American Ceramic Society, Vol 55, $\mathrm{n}$ 10, 1972, pp 514-519

${ }^{24}$ B.V. Bondarenko, E.P. Ostapchenko, B.M. Tsarev, “Thermionic Properties of Alkali Metal Tungstates”, Radiotekh. Elektron, Vol 5, 1960, pp 1246-1253

${ }^{25}$ H. Kingdon, I. Langmuir, "The Removal of Thorium from the Surface of a Thoriated Tungsten Filament by Positive Ion Bombardment”, Physical review, 1923

${ }^{26}$ I. Langmuir, “The Nature Of Adsorbed Films of Caesium on Tungsten”, Physical review, vol 43, 1933

${ }^{27}$ I. Langmuir, "The Electron Emission from Thoriated Tungsten Filaments", Physical review, vol 22, 1923

${ }^{28}$ I. Langmuir, K.H. Kingdon, “Thermionic Effects Caused by Vapours of Alkali Metals”, Proceeding of the Royal Society of London, vol 107, no 741, 1925, pp 61-79

${ }^{29}$ J.B. Taylor, I. Langmuir, “The Evaporation of Atoms, Ions and Electrons from Caesium Films on Tungsten”, Physical Review, vol 44, n 6, 1933

${ }^{30}$ R.A. Lipeles, H.K.A Kan, "Chemical Stability of Barium Calcium Aluminate Dispenser Cathode Impregnants", Application of Surface Science 16, 1983, pp. 189-206

${ }^{31}$ Wolten G.M., "An Appraisal of the Ternary System BaO-CaO- $\mathrm{Al}_{2} \mathrm{O}_{3}$ ”, SD-TR-80-67, Space Division, Air Force System Command, Los Angeles, October 1980

${ }^{32}$ D.M. Goebel, K. Jameson, I. Katz, I.G. Mikellides, "Energetic Ion Production and Keeper Erosion in Hollow Cathode Discharges", IEPC-2005-266, 29 $9^{\text {th }}$ International Electric Propulsion Conference, Princeton, 31 October - 4 November , 2005

${ }^{33}$ K. Jameson, D.M. Goebel, RM. Watkins, "Hollow Cathode and Thruster Discharge Chamber Plasma Measurements", IEPC-2005-269, $29^{\text {th }}$ International Electric Propulsion Conference, Princeton, 31 October - 4 November , 2005

${ }^{34}$ P. Silvestroni, "Fondamenti di Chimica”, Veschi Editore, Roma, 1974

${ }^{35}$ P. Sigmund, ”Theory of Sputtering. I. Sputtering Yield of Amorphous and Polycrystalline Targets”, Phys. Rev. 184, 383 416 (1969)

${ }^{36}$ D.E. Sigfried, P.J. Wilbur “A Model for Mercury Orificed Hollow Cathodes: Theory and Experiment” AIAA Journal, Vol. 22, No. 10, October 1984 